\title{
Superspace formulation of massive half-integer superspin
}

\author{
Konstantinos Koutrolikos \\ Brown Theoretical Physics Center, \\ Box S, 340 Brook Street, Barus, Providence, RI 02912, U.S.A. \\ Department of Physics, Brown University, \\ Box 1843, 182 Hope Street, Barus \& Holley, Providence, RI 02912, U.S.A. \\ E-mail: konstantinos_koutrolikos@brown.edu
}

ABSTRACT: An explicit form for the Lagrangian of a massive arbitrary half-integer superspin $\mathrm{Y}=s+1 / 2$ supermultiplet is obtained in $4 \mathrm{D}, \mathcal{N}=1$ superspace. This is accomplished by the introduction of a tower of pairs of auxiliary superfields of increasing rank which are required to vanish on-shell for free theories. In the massless limit almost all auxiliary superfields decouple except one, which plays the role of compensator as required by the emergent gauge redundancy of the Lagrangian description of the massless theory. The number of off-shell degrees of freedom carried by the theory is $\frac{8}{3}(s+1)\left(4 s^{2}+11 s+3\right)$. For $s=1$ our results are in agreement with those obtained in [1].

KEYwords: Higher Spin Symmetry, Superspaces, Supersymmetric Effective Theories ArXiv EPrint: 2012.12225

Dedicated to S. James Gates Jr. on the occasion of his 70th birthday 


\section{Contents}

1 Introduction 1

2 Massive and massless irreducible representations 2

3 Arbitrary half integer superspin supermultiplet action 4

3.1 Linearized massive supergravity and $s$-th level auxiliary superfields $\quad 9$

$3.2 \mathrm{Y}=5 / 2$ supermultiplet and $(s-1)$-th level auxiliary superfields 11

$\begin{array}{lll}3.3 & \text { Vanishing of } q \text {-th level auxiliary superfields } & 16\end{array}$

4 Off-shell degrees of freedom $\quad 18$

$\begin{array}{lll}5 & \text { Summary } & 19\end{array}$

\section{Introduction}

Since the discovery of supersymmetry in the late 1960's and early 1970 's ${ }^{1}$ there has been tremendous progress in the development of supersymmetric theories which undoubtedly influenced a big part of theoretical physics. For 4D higher spin theories, early developments led to the supersymmetric extension of free, massless, irreducible representations with on-shell supersymmetry and their Lagrangian description $[4,5]$. The off-shell, superspace description of such representations was found first in [6-8] and later developments are [9-11]. Furthermore, various consistent interactions among massless higher spin supermultiplets have been discovered recently [12-22].

However, after five decades of intensive investigations, the seemingly simple question of finding the superspace action principle for free, massive, arbitrary superspin, irreducible representation of $4 \mathrm{D}, \mathcal{N}=1$ super-Poincaré group still remains unanswered. This is a very important and necessary first step in order to even consider exploring manifestly supersymmetric interactions among massive arbitrary spin supermultiplets. The non-supersymmetric Lagrangian description of massive irreducible representations of the Poincaré group was found in $[23,24]$ almost forty years after the theory of higher spins was first undertaken by Dirac [25]. It was demonstrated that massive higher spins carry a very interesting and rich off-shell structure, because they require a (double) tower of auxiliary bosonic (fermionic) fields of increasing rank.

For the case of supersymmetry such a description and understanding of the off-shell structure of arbitrary higher spin supermultiplet is lacking, nevertheless there has been some progress towards that direction. In [26-30] a Lagrangian description of on-shell massive higher spin supermultiplets was found. This description follows the viewpoint of

\footnotetext{
${ }^{1}$ The dramatic story of the discovery of supersymmetry and its early years can be found in $[2,3]$.
} 
perceiving the on-shell massive spin degrees of freedom as a collection of on-shell massless helicities ${ }^{2}$ and thus, by stitching together a tower of on-shell, massless supermultiplets with increasing spin, one can find a description for the on-shell massive higher spin supermultiplet. However, because the supermultiplets considered only have on-shell supersymmetry, this approach provides no clues regarding the additional auxiliary structures a manifestly supersymmetry description may require. Manifestly supersymmetric descriptions of massive irreducible representations do exist [1,31-35] but unfortunately not for higher spin supermultiplets.

Nevertheless, the theory constructed in [1] corresponds to the massive extension of one of the formulations of linearized supergravity which can be extended to arbitrary halfinteger superspin theories. In this paper we construct the superspace Lagrangian for a $4 \mathrm{D}, \mathcal{N}=1$ super-Poincaré irreducible representation of mass $m$ and arbitrary superspin $\mathrm{Y}=s+1 / 2$. We find that in addition to the real bosonic superfield $H_{\alpha(s) \dot{\alpha}(s)}$ which carries the propagating, on-shell degrees of freedom of this representation, the superspace Lagrangian description requires a tower of pairs of auxiliary, fermionic superfields $\chi_{\alpha(q) \dot{\alpha}(q-1)}, u_{\alpha(q) \dot{\alpha}(q-1)}$ with $1 \leq q \leq s$. This is in agreement with the results found in [1] for the special case of $s=1$ and also is consistent with the structures required by the non-supersymmetric results $[23,24]$. The explicit form of the Lagrangian is found by demanding that all these auxiliary superfields vanish on-shell, in the free theory limit and the appropriate constraints on $H_{\alpha(s) \dot{\alpha}(s)}$ are generated in order to describe the irreducible representation $\mathrm{Y}=s+1 / 2$.

The paper is organized as follows. In section 2 we review basic features of irreducible representations of the $4 \mathrm{D}, \mathcal{N}=1$ super-Poincaré group. In section 3 we construct the superspace action that provides the off-shell description of these irreducible representations for the arbitrary half-integer superspin supermultiplet. In section 4 we illustrate the richness of the off-shell structure, by counting the number of off-shell degrees of freedom of this theory.

\section{Massive and massless irreducible representations of 4D super-Poincaré}

Often in physics we consider some symmetry (super) group and then ask about its (irreducible) representations. For the majority of the cases, the answer about their existence and their classification almost certainly already exists somewhere in mathematical literature. However, for many applications in physics this is not enough, because we do not just blindly look for any representations but for locally realizable representations that can have a (super) field theoretic description. This tension between knowing the representation and realize it in a field theoretic framework is responsible for the richness of many theories.

In this case we consider the $4 \mathrm{D}, \mathcal{N}=1$ super-Poincaré group but in order to have a finite number of propagating degrees of freedom we focus on its stabilizer, the super-

\footnotetext{
${ }^{2}$ The $2 s+1$ on-shell degrees of freedom of a massive spin $j=s$ can be viewed as a collection of the $-j,+j$ on-shell helicities for massless spins $j=0,1, \ldots, s$ if $s$ is integer or $j=1 / 2,3 / 2, \ldots, s$ if $s$ is half-integer. Moreover, if one trust this analogy to hold off-shell, then one will precisely discover the (double) tower of auxiliary symmetric (spinor) tensor fields appearing in the off-shell Lagrangian description of [23, 24].
} 
Little group. ${ }^{3}$ The diagonalization of its two Casimir operators and its Cartan subalgebra determines $(i)$ the type of superfield (number of indices and their symmetries) which realizes the representation and ( $i i)$ the type of constraints it must satisfy in order to be irreducible. For a massive, half-integer superspin $(\mathrm{Y}=s+1 / 2)$ representation one must consider a real bosonic superfield $H_{\alpha(s) \dot{\alpha}(s)}$, with $s$ dotted and undotted indices which are independently symmetrized and satisfies the following conditions

$$
\square H_{\alpha(s) \dot{\alpha}(s)}=m^{2} H_{\alpha(s) \dot{\alpha}(s)}, \mathrm{D}^{\alpha_{s}} H_{\alpha(s) \dot{\alpha}(s)}=0 .
$$

These constraints kill most of the components of superfield $H_{\alpha(s) \dot{\alpha}(s)}$ but allow the propagation of the physical degrees of freedom of four massive spins, $j=s+1, j=s+1 / 2$, $j=s+1 / 2, j=s$. Describing an irreducible representation this way is an on-shell statement, since it refers to the physical degrees of freedom. A natural question to ask is what is the off-shell description of this representation. This can be given in terms of an action which will generate equations of motion that give raise to the desired constraints. In this paper, we will answer this question for the case of an arbitrary half-integer superspin supermultiplet described by (2.1). We will show that the answer includes a hierarchy of pairs of auxiliary, fermionic, superfields $\chi_{\alpha(q) \dot{\alpha}(q-1)}, u_{\alpha(q) \dot{\alpha}(q-1)}$ for $q=1,2, \ldots, s$.

For a massless, half-integer superspin supermultiplet, the on-shell propagating degrees of freedom are described by the superfield strength $W_{\alpha(2 s+1)}$. This is a chiral, fermionic superfield with $2 s+1$ symmetrized undotted indices which must satisfy the constraints

$$
\mathrm{D}^{\alpha_{2 s+1}} W_{\alpha(2 s+1)}=0, \overline{\mathrm{D}}_{\dot{\beta}} W_{\alpha(2 s+1)}=0 .
$$

This is analogous to the description of the massless spin one representation by the field strength $F_{m n}$. It is the field strength that carries the physical degrees of freedom, since if we do an experiment we will measure the electric and magnetic fields. $F_{m n}$ must satisfy on-shell the constraints $\partial^{m} F_{m n}=0, \partial_{[k} F_{m n]}=0$. One of them we call the dynamical equations of motion and the other we call the Bianchi identity. In order to construct the off-shell, Lagrangian description of the theory we solve the Bianchi identity in terms of a gauge vector field. The fact that the off-shell description of the theory is given in terms of the gauge vector field, allows for a smooth transition between the Lagrangian description of a massive spin one (Proca action) and the Lagrangian description of a massless spin one (Maxwell's theory) by taking the massless limit of the Proca action. The discontinuity on the onshell degrees of freedom is recovered from the emergence of the redundancy of the vector field in the massless limit. This approach can be applied to the case of massless irreducible representations of the super-Poincaré group (see [9]). Briefly, the second constraint in (2.2) can be solved by expressing superfield strength $W_{\alpha(2 s+1)}$ in terms of a real prepotential superfield which has the same index structure as the superfield that describes the massive theory (and thus making the transition from massive action to massless one smooth)

$$
W_{\alpha(2 s+1)} \propto \overline{\mathrm{D}}^{2} \mathrm{D}_{\left(\alpha_{2 s+1}\right.} \partial_{\alpha_{2 s}}{ }^{\dot{\alpha}_{s}} \ldots \partial_{\alpha_{s+1}}{ }^{\dot{\alpha}_{1}} H_{\alpha(s)) \dot{\alpha}(s)} .
$$

\footnotetext{
${ }^{3}$ For a detailed review see $[9,36-41]$.
} 
The prepotential $H_{\alpha(s) \dot{\alpha}(s)}$, is now a gauge superfield because it acquires a redundancy, which leaves the superfield strength invariant

$$
\delta H_{\alpha(s) \dot{\alpha}(s)}=\frac{1}{s !} \mathrm{D}_{\left(\alpha_{s}\right.} \bar{L}_{\alpha(s-1)) \dot{\alpha}(s)}-\frac{1}{s !} \overline{\mathrm{D}}_{\left(\dot{\alpha}_{s}\right.} L_{\alpha(s) \dot{\alpha}(s-1))} .
$$

Using the above redundancy as a guiding principle, one can find the superspace Lagrangian description for this supermultiplet. The result is that there are two different off-shell formulations of the same theory based on $(2.4)[6,9,11]$. Both of them require the presence of an additional, unconstrained, compensating superfield. The one relevant for our discussion ${ }^{4}$ is the non-minimal description given by the following action principle

$$
\begin{aligned}
S_{(\mathrm{Y}=s+1 / 2)}=\int & d^{8} z H^{\alpha(s) \dot{\alpha}(s)} \mathrm{D}^{\gamma} \overline{\mathrm{D}}^{2} \mathrm{D}_{\gamma} H_{\alpha(s) \dot{\alpha}(s)} \\
& -2 H^{\alpha(s) \dot{\alpha}(s)} \overline{\mathrm{D}}_{\dot{\alpha}_{s}} \mathrm{D}^{2} \chi_{\alpha(s) \dot{\alpha}(s-1)}+c . c . \\
& -\frac{s+1}{s} \chi^{\alpha(s) \dot{\alpha}(s-1)} \mathrm{D}^{2} \chi_{\alpha(s) \dot{\alpha}(s-1)}+c . c . \\
& +2 \chi^{\alpha(s) \dot{\alpha}(s-1)} \mathrm{D}_{\alpha_{s}} \overline{\mathrm{D}}^{\dot{\alpha}_{s}} \bar{\chi}_{\alpha(s-1) \dot{\alpha}(s)}
\end{aligned}
$$

where the unconstrained compensator has the redundancy

$$
\delta \chi_{\alpha(s) \dot{\alpha}(s-1)}=\overline{\mathrm{D}}^{2} L_{\alpha(s) \dot{\alpha}(s-1)}+\mathrm{D}^{\alpha_{s+1}} \Lambda_{\alpha(s+1) \dot{\alpha}(s-1)} .
$$

The equations of motion of the above action will generate the desired condition (2.2) and thus allow only the propagation of the helicities of spins $j=s+1$ and $j=s+1 / 2$.

\section{Arbitrary half integer superspin supermultiplet action}

The massive extension of $S_{(\mathrm{Y}=s+1 / 2)}$, denoted as $S_{(m, \mathrm{Y}=s=1 / 2)}$, must be such that

$$
\begin{aligned}
\lim _{m \rightarrow 0}\left[S_{(m, \mathrm{Y}=s+1 / 2)}\right] & =S_{(\mathrm{Y}=s+1 / 2)}+S_{(\text {decoupled sector })}, \\
\delta S_{(m, \mathrm{Y}=s+1 / 2)} & =0 \Rightarrow \square H_{\alpha(s) \dot{\alpha}(s)}=m^{2} H_{\alpha(s) \dot{\alpha}(s)}, \quad \mathrm{D}^{\alpha_{s}} H_{\alpha(s) \dot{\alpha}(s)}=0 .
\end{aligned}
$$

For simple cases, like the vector supermultiplet $(s=0)$ the $S_{\text {(decoupled sector) }}$ is trivially zero. However, in general we can allow such term. As an example, consider the theory of linearized massive supergravity developed in [1]. It requires the presence of an additional auxiliary superfield $u_{\alpha}$ which in the massless limit decoupled from the theory. Similar behavior is demonstrated in $[23,24]$ were the massless limit of the theory generates a non trivial decoupled sector which can be ignored from the view point of the massless theory. Condition (3.1) dictates that the most general interaction between the auxiliary superfields in $S_{\text {(decoupled sector) }}$ and the superfields of massless theory must depend on the mass parameter. Finally, the various numerical coefficients will be fixed by demanding that all auxiliary superfields vanish on-shell and condition (3.2) is satisfied. This will fix the on-shell spectrum of the theory to be the correct irreducible representation.

\footnotetext{
${ }^{4}$ We follow the conventions of Superspace [37].
} 
Motivated from the results in [23, 24] and [1], we propose the following coupling scheme:

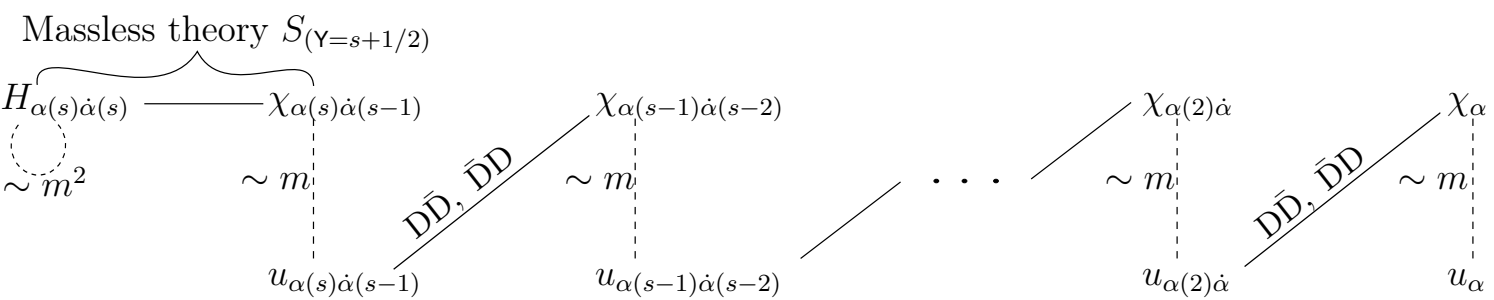

Starting from the massless action $S_{(\mathrm{Y}=s+1 / 2)}$ we introduce a double tower of auxiliary superfields $\chi_{\alpha(q) \dot{\alpha}(q-1)}, u_{\alpha(q) \dot{\alpha}(q-1)}$ equipped with two types of interactions. The $q$-th level superfields $\chi^{(q)}$ and $u^{(q)}$ interact via a mass term (vertical dashed lines). This type of interaction dissolves at the massless limit. The second type of interaction is between the $q$-th level superfield $u^{(q)}$ and the $(q-1)$-th level $\chi^{(q-1)}$ superfield (solid diagonal lines) and is persistent, in the sense that it survives the massless limit. Furthermore, in compliance with the preliminary results of [1], superfield $H_{\alpha(s) \dot{\alpha}(s)}$ has its own mass term as expected, but the auxiliary superfields $\chi^{(q)}, u^{(q)}$ do not have their own mass term. The consistency of this scheme with the massless limit is automatic, because the first vertical dashed line will break, decoupling the entire $\chi, u$ sector, except $\chi^{(s)}$ and thus recovers the correct massless action $S_{(\mathrm{Y}=s+1 / 2)}$. Also it is easy to check the consistency with the $s=1$ results of [1]. In that case there is only one level $(q=1)$ and thus, only the mass interaction between $\chi_{\alpha}$ and $u_{\alpha}$ participates, exactly as found. The most general action that reflects this coupling scheme is

$$
\begin{aligned}
S_{(m, \mathrm{Y}=s+1 / 2)}=\int d^{8} z\{ & H^{\alpha(s) \dot{\alpha}(s)} \mathrm{D}^{\gamma} \overline{\mathrm{D}}^{2} \mathrm{D}_{\gamma} H_{\alpha(s) \dot{\alpha}(s)}+m^{2} H^{\alpha(s) \dot{\alpha}(s)} H_{\alpha(s) \dot{\alpha}(s)} \\
& -2 H^{\alpha(s) \dot{\alpha}(s)} \overline{\mathrm{D}}_{\dot{\alpha}_{s}} \mathrm{D}^{2} \chi_{\alpha(s) \dot{\alpha}(s-1)}+c . c . \\
& -\frac{s+1}{s} \chi^{\alpha(s) \dot{\alpha}(s-1)} \mathrm{D}^{2} \chi_{\alpha(s) \dot{\alpha}(s-1)}+c . c . \\
& +2 \chi^{\alpha(s) \dot{\alpha}(s-1)} \mathrm{D}_{\alpha_{s}} \overline{\mathrm{D}}^{\dot{\alpha}_{s}} \bar{\chi}_{\alpha(s-1) \dot{\alpha}(s)} \\
& +m \chi^{\alpha(s) \dot{\alpha}(s-1)} u_{\alpha(s) \dot{\alpha}(s-1)}+c . c . \\
& +c_{1}^{(s)} u^{\alpha(s) \dot{\alpha}(s-1)} \overline{\mathrm{D}}^{2} u_{\alpha(s) \dot{\alpha}(s-1)}+c . c . \\
& +c_{2}^{(s)} u^{\alpha(s) \dot{\alpha}(s-1)} \mathrm{D}^{2} u_{\alpha(s) \dot{\alpha}(s-1)}+c . c . \\
& +c_{3}^{(s)} u^{\alpha(s) \dot{\alpha}(s-1)} \overline{\mathrm{D}}^{\dot{\alpha}_{s}} \mathrm{D}_{\alpha_{s}} \bar{u}_{\alpha(s-1) \dot{\alpha}(s)} \\
& +c_{4}^{(s)} u^{\alpha(s) \dot{\alpha}(s-1)} \mathrm{D}_{\alpha_{s}} \overline{\mathrm{D}}^{\dot{\alpha}_{s}} \bar{u}_{\alpha(s-1) \dot{\alpha}(s)}
\end{aligned}
$$




$$
\begin{aligned}
&+\sum_{q=1}^{s-1}\left[u^{\alpha(q+1) \dot{\alpha}(q)}(\right.\left.b_{1}^{(q+1)} \overline{\mathrm{D}}_{\dot{\alpha}_{q}} \mathrm{D}_{\alpha_{q+1}} \chi_{\alpha(q) \dot{\alpha}(q-1)}+b_{2}^{(q+1)} \mathrm{D}_{\alpha_{q+1}} \overline{\mathrm{D}}_{\dot{\alpha}_{q}} \chi_{\alpha(q) \dot{\alpha}(q-1)}\right)+c . c . \\
&+d_{1}^{(q)} \chi^{\alpha(q) \dot{\alpha}(q-1)} \overline{\mathrm{D}}^{2} \chi_{\alpha(q) \dot{\alpha}(q-1)}+c . c . \\
&+d_{2}^{(q)} \chi^{\alpha(q) \dot{\alpha}(q-1)} \mathrm{D}^{2} \chi_{\alpha(q) \dot{\alpha}(q-1)}+c . c . \\
&+d_{3}^{(q)} \chi^{\alpha(q) \dot{\alpha}(q-1)} \overline{\mathrm{D}}^{\dot{\alpha}_{q}} \mathrm{D}_{\alpha_{q}} \bar{\chi}_{\alpha(q-1) \dot{\alpha}(q)} \\
&+d_{4}^{(q)} \chi^{\alpha(q) \dot{\alpha}(q-1)} \mathrm{D}_{\alpha_{q}} \overline{\mathrm{D}}^{\dot{\alpha}_{q}} \bar{\chi}_{\alpha(q-1) \dot{\alpha}(q)} \\
&+m \chi^{\alpha(q) \dot{\alpha}(q-1)} u_{\alpha(q) \dot{\alpha}(q-1)}+c . c . \\
&+c_{1}^{(q)} u^{\alpha(q) \dot{\alpha}(q-1)} \overline{\mathrm{D}}^{2} u_{\alpha(q) \dot{\alpha}(q-1)}+c . c . \\
&+c_{2}^{(q)} u^{\alpha(q) \dot{\alpha}(q-1)} \mathrm{D}^{2} u_{\alpha(q) \dot{\alpha}(q-1)}+c . c . \\
&+c_{3}^{(q)} u^{\alpha(q) \dot{\alpha}(q-1)} \overline{\mathrm{D}}^{\dot{\alpha}_{q}} \mathrm{D}_{\alpha_{q}} \bar{u}_{\alpha(q-1) \dot{\alpha}(q)} \\
&\left.\left.+c_{4}^{(q)} u^{\alpha(q) \dot{\alpha}(q-1)} \mathrm{D}_{\alpha_{q}} \overline{\mathrm{D}}^{\dot{\alpha}_{q}} \bar{u}_{\alpha(q-1) \dot{\alpha}(q)}\right]\right\} .
\end{aligned}
$$

The coefficient of the mass interaction between $\chi^{(q)}$ and $u^{(q)}$ can be set to one by adjusting the normalization of $u^{(q)}$. Similarly, one of the two $b^{(q+1)}$ coefficients can also be set to one by adjusting the normalization of $\chi^{(q)}$. The equations of motion generated by the above action are

$$
\begin{aligned}
\mathcal{E}_{\alpha(s) \dot{\alpha}(s)}^{(H)}= & 2 \mathrm{D}^{\gamma} \overline{\mathrm{D}}^{2} \mathrm{D}_{\gamma} H_{\alpha(s) \dot{\alpha}(s)}+2 m^{2} H_{\alpha(s) \dot{\alpha}(s)}-\frac{2}{s !} \overline{\mathrm{D}}_{\left(\dot{\alpha}_{s}\right.} \mathrm{D}^{2} \chi_{\alpha(s) \dot{\alpha}(s-1))} \\
& +\frac{2}{s !} \mathrm{D}_{\left(\alpha_{s}\right.} \overline{\mathrm{D}}^{2} \bar{\chi}_{\alpha(s-1)) \dot{\alpha}(s)} \\
\mathcal{E}_{\alpha(s) \dot{\alpha}(s-1)}^{(\chi, s)}= & -2 \mathrm{D}^{2} \overline{\mathrm{D}}^{\dot{\alpha}_{s}} H_{\alpha(s) \dot{\alpha}(s)}-2 \frac{s+1}{s} \mathrm{D}^{2} \chi_{\alpha(s) \dot{\alpha}(s-1)} \\
& +\frac{2}{s !} \mathrm{D}_{\left(\alpha_{s}\right.} \overline{\mathrm{D}}^{\dot{\alpha}_{s}} \bar{\chi}_{\alpha(s-1)) \dot{\alpha}(s)}+m u_{\alpha(s) \dot{\alpha}(s-1)} \\
\mathcal{E}_{\alpha(s) \dot{\alpha}(s-1)}^{(u, s)} & 2 c_{1}^{(s)} \overline{\mathrm{D}}^{2} u_{\alpha(s) \dot{\alpha}(s-1)}+2 c_{2}^{(s)} \mathrm{D}^{2} u_{\alpha(s) \dot{\alpha}(s-1)}+\frac{c_{3}^{(s)}}{s !} \overline{\mathrm{D}}^{\dot{\alpha}_{s}} \mathrm{D}_{\left(\alpha_{s}\right.} \bar{u}_{\alpha(s-1)) \dot{\alpha}(s)} \\
& +\frac{c_{4}^{(s)}}{s !} \mathrm{D}_{\left(\alpha_{s}\right.} \overline{\mathrm{D}}^{\dot{\alpha}_{s}} \bar{u}_{\alpha(s-1)) \dot{\alpha}(s)}+m \chi_{\alpha(s) \dot{\alpha}(s-1)}+\frac{b_{1}^{(s)}}{s !(s-1) !} \overline{\mathrm{D}}_{\left(\dot{\alpha}_{s-1}\right.} \mathrm{D}_{\left(\alpha_{s}\right.} \chi_{\alpha(s-1)) \dot{\alpha}(s-2))} \\
& +\frac{b_{2}^{(s)}}{s !(s-1) !} \mathrm{D}_{\left(\alpha_{s}\right.} \overline{\mathrm{D}}_{\left(\dot{\alpha}_{s-1}\right.} \chi_{\alpha(s-1)) \dot{\alpha}(s-2))}
\end{aligned}
$$

and

$$
\begin{aligned}
\mathcal{E}_{\alpha(q) \dot{\alpha}(q-1)}^{(\chi, q)}= & -b_{1}^{(q+1)} \mathrm{D}^{\alpha_{q+1}} \overline{\mathrm{D}}^{\dot{\alpha}_{q}} u_{\alpha(q+1) \dot{\alpha}(q)}-b_{2}^{(q+1)} \overline{\mathrm{D}}^{\dot{\alpha}_{q}} \mathrm{D}^{\alpha_{q+1}} u_{\alpha(q+1) \dot{\alpha}(q)} \\
& +2 d_{1}^{(q)} \overline{\mathrm{D}}^{2} \chi_{\alpha(q) \dot{\alpha}(q-1)}+2 d_{2}^{(q)} \mathrm{D}^{2} \chi_{\alpha(q) \dot{\alpha}(q-1)}+\frac{d_{3}^{(q)}}{q !} \overline{\mathrm{D}}^{\dot{\alpha}_{q}} \mathrm{D}_{\left(\alpha_{q}\right.} \bar{\chi}_{\alpha(q-1)) \dot{\alpha}(q)} \\
& +\frac{d_{4}^{(q)}}{q !} \mathrm{D}_{\left(\alpha_{q}\right.} \overline{\mathrm{D}}^{\dot{\alpha}_{q}} \bar{\chi}_{\alpha(q-1)) \dot{\alpha}(q)}+m u_{\alpha(q) \dot{\alpha}(q-1)}
\end{aligned}
$$




$$
\begin{aligned}
\mathcal{E}_{\alpha(q) \dot{\alpha}(q-1)}^{(u, q)}= & 2 c_{1}^{(q)} \overline{\mathrm{D}}^{2} u_{\alpha(q) \dot{\alpha}(q-1)}+2 c_{2}^{(q)} \mathrm{D}^{2} u_{\alpha(q) \dot{\alpha}(q-1)}+\frac{c_{3}^{(q)}}{q !} \overline{\mathrm{D}}^{\dot{\alpha}_{q}} \mathrm{D}_{\left(\alpha_{q}\right.} \bar{u}_{\alpha(q-1)) \dot{\alpha}(q)} \\
& +\frac{c_{4}^{(q)}}{q !} \mathrm{D}_{\left(\alpha_{q}\right.} \overline{\mathrm{D}}^{\dot{\alpha}_{q}} \bar{u}_{\alpha(q-1)) \dot{\alpha}(q)}+m \chi_{\alpha(q) \dot{\alpha}(q-1)} \\
& +\frac{b_{1}^{(q)}}{q !(q-1) !} \overline{\mathrm{D}}_{\left(\dot{\alpha}_{q-1}\right.} \mathrm{D}_{\left(\alpha_{q}\right.} \chi_{\alpha(q-1)) \dot{\alpha}(q-2))} \\
& +\frac{b_{2}^{(q)}}{q !(q-1) !} \mathrm{D}_{\left(\alpha_{q}\right.} \overline{\mathrm{D}}_{\left(\dot{\alpha}_{q-1}\right.} \chi_{\alpha(q-1)) \dot{\alpha}(q-2))}
\end{aligned}
$$

for $q=1,2, \ldots, s-1$. Our strategy is to use these equations of motion with an appropriate choice of coefficients in order to show that on-shell all auxiliary superfields vanish and we dynamically generate constraints $(2.1)$ :

$$
\begin{aligned}
& u_{\alpha}=0 \Rightarrow \chi_{\alpha}=0 \Rightarrow \cdots \Rightarrow u_{\alpha(q) \dot{\alpha}(q-1)}=0 \Rightarrow \chi_{\alpha(q) \dot{\alpha}(q-1)}=0 \Rightarrow \ldots \\
& \Rightarrow u_{\alpha(s) \dot{\alpha}(s-1)}=0 \Rightarrow \chi_{\alpha(s) \dot{\alpha}(s-1)}=0 \Rightarrow \mathrm{D}^{\alpha_{s}} H_{\alpha(s) \dot{\alpha}(s)}=0, \quad \square H_{\alpha(s) \dot{\alpha}(s)}=m^{2} H_{\alpha(s) \dot{\alpha}(s)} .
\end{aligned}
$$

We will do that in steps and recursively. For example, if we assume that $u^{(r)}=\chi^{(r)}=0$ for $r=1,2, \ldots, s$ then we can easily generate (2.1). If $u^{(s)}=\chi^{(s)}=0$ we get via (3.5) that on-shell $\mathrm{D}^{2} \overline{\mathrm{D}}^{\dot{\alpha}_{s}} H_{\alpha(s) \dot{\alpha}(s)}=0$. This will lead to $\mathrm{D}^{\alpha_{s}} H_{\alpha(s) \dot{\alpha}(s)}=0$ based on $\mathrm{D}^{\alpha_{s}} \mathcal{E}_{\alpha(s) \dot{\alpha}(s)}^{(H)}=0$ and $\mathcal{E}_{\alpha(s) \dot{\alpha}(s)}^{(H)}=0$ will give $\square H_{\alpha(s) \dot{\alpha}(s)}=m^{2} H_{\alpha(s) \dot{\alpha}(s)}$. The next step is to assume that $u^{(r)}=\chi^{(r)}=0$ for $r=1,2, \ldots, s-1$ and show that there is a choice of $c^{(s)}$ coefficients such that $u^{(s)}, \chi^{(s)}$ both vanish on-shell. We will show that this approach can be iterated until we reach the bottom of the sequence (3.9) and in the process we determine all coefficients. In every iteration we assume that all auxiliary superfields up to some level can be set to zero and then prove that there is an appropriate choice of coefficients that will make the next level auxiliary superfields vanish too.

Because the auxiliary superfields are required to vanish on-shell, one is allowed to consider a linear redefinition of the auxiliary superfields $\hat{u}^{(q)}=A u^{(q)}+B \chi^{(q)}$, $\hat{\chi}^{(q)}=C u^{(q)}+D \chi^{(q)}$ without affecting the outcome. Besides this redefinition being invertible $(A D-C B \neq 0)$, the coefficients $(A, B, C, D)$ are arbitrary and can be used to fix any four, q-level coefficients in the action. This freedom has already being used in the ansatz (3.3).

A tool that will be used often in this method is the following. Notice that $\chi^{(q)}$ superfield appears algebraically in $\mathcal{E}^{(u, q)}$. Hence, we can use this to eliminate all $\chi^{(q)}$ dependence in $\mathcal{E}^{(\chi, q)}$, for all values of $q$. One way of doing that is to define the following quantity:

$$
\begin{aligned}
\mathcal{I}_{\alpha(q) \dot{\alpha}(q-1)}^{(q)}= & m \mathcal{E}_{\alpha(q) \dot{\alpha}(q-1)}^{(\chi, q)}-2 d_{1}^{(q)} \overline{\mathrm{D}}^{2} \mathcal{E}_{\alpha(q) \dot{\alpha}(q-1)}^{(u, q)}-2 d_{2}^{(q)} \mathrm{D}^{2} \mathcal{E}_{\alpha(q) \dot{\alpha}(q-1)}^{u, q)} \\
& -\frac{d_{3}^{(q)}}{q !} \overline{\mathrm{D}}^{\dot{\alpha}_{q}} \mathrm{D}_{\left(\alpha_{q}\right.} \overline{\mathcal{E}}_{\alpha(q-1)) \dot{\alpha}(q)}^{(u, q)}-\frac{d_{4}^{(q)}}{q !} \mathrm{D}_{\left(\alpha_{q}\right.} \overline{\mathrm{D}}^{\dot{\alpha}_{q}} \overline{\mathcal{E}}_{\alpha(q-1)) \dot{\alpha}(q)}^{(u, q)}
\end{aligned}
$$


Plugging in equations (3.7) and (3.8) we find that

$$
\begin{aligned}
& \mathcal{I}_{\alpha(q) \dot{\alpha}(q-1)}^{(q)}=-m b_{1}^{(q+1)} \mathrm{D}^{\alpha_{q+1}} \overline{\mathrm{D}}^{\dot{\alpha}_{q}} u_{\alpha(q+1) \dot{\alpha}(q)}-m b_{2}^{(q+1)} \overline{\mathrm{D}}^{\dot{\alpha}_{q}} \mathrm{D}^{\alpha_{q+1}} u_{\alpha(q+1) \dot{\alpha}(q)} \\
& +m^{2} u_{\alpha(q) \dot{\alpha}(q-1)}+\left\{d_{3}^{(q)} c_{3}^{(q)}\right\} \mathrm{D}^{\gamma} \overline{\mathrm{D}}^{2} \mathrm{D}_{\gamma} u_{\alpha(q) \dot{\alpha}(q-1)} \\
& +\left\{-4 d_{1}^{(q)} c_{2}^{(q)}-d_{3}^{(q)} c_{4}^{(q)}\right\} \overline{\mathrm{D}}^{2} \mathrm{D}^{2} u_{\alpha(q) \dot{\alpha}(q-1)}+\left\{-4 d_{2}^{(q)} c_{1}^{(q)}-d_{4}^{(q)} c_{3}^{(q)}\right\} \mathrm{D}^{2} \overline{\mathrm{D}}^{2} u_{\alpha(q) \dot{\alpha}(q-1)} \\
& +\left\{-2 d_{1}^{(q)} c_{4}^{(q)}-2 d_{3}^{(q)} c_{2}^{(q)}\right\} \frac{1}{q !} \overline{\mathrm{D}}^{2} \mathrm{D}_{\left(\alpha_{q}\right.} \overline{\mathrm{D}}^{\dot{\alpha}_{q}} \bar{u}_{\alpha(q-1)) \dot{\alpha}(q)} \\
& +\left\{-2 d_{2}^{(q)} c_{3}^{(q)}-2 d_{4}^{(q)} c_{1}^{(q)}\right\} \frac{1}{q !} \mathrm{D}^{2} \overline{\mathrm{D}}^{\dot{\alpha}_{q}} \mathrm{D}_{\left(\alpha_{q}\right.} \bar{u}_{\alpha(q-1)) \dot{\alpha}(q)} \\
& +\left\{d_{3}^{(q)} c_{4}^{(q)}-d_{4}^{(q)}\left(\frac{q+1}{q} c_{4}^{(q)}-c_{3}^{(q)}\right)\right\} \frac{1}{q !} \mathrm{D}_{\left(\alpha_{q}\right.} \overline{\mathrm{D}}^{2} \mathrm{D}^{\beta} u_{\beta \alpha(q-1)) \dot{\alpha}(q-1)} \\
& +\left\{\frac{q-1}{q} d_{3}^{(q)} c_{3}^{(q)}\right\} \frac{1}{(q-1) !} \overline{\mathrm{D}}_{\left(\dot{\alpha}_{q-1}\right.} \mathrm{D}^{2} \overline{\mathrm{D}}^{\dot{\beta}} u_{\alpha(q) \dot{\beta} \dot{\alpha}(q-2))} \\
& +\left\{-\frac{q-1}{q} d_{4}^{(q)} c_{3}^{(q)}\right\} \frac{1}{q !(q-1) !} \mathrm{D}_{\left(\alpha_{q}\right.} \overline{\mathrm{D}}_{\left(\dot{\alpha}_{q-1}\right.} \mathrm{D}^{\beta} \overline{\mathrm{D}}^{\dot{\beta}} u_{\beta \alpha(q-1)) \dot{\beta} \dot{\alpha}(q-2))} \\
& +\left\{-\frac{q-1}{q} d_{3}^{(q)} c_{4}^{(q)}\right\} \frac{1}{q !(q-1) !} \overline{\mathrm{D}}_{\left(\dot{\alpha}_{q-1}\right.} \mathrm{D}_{\left(\alpha_{q}\right.} \overline{\mathrm{D}}^{\dot{\beta}} \mathrm{D}^{\beta} u_{\beta \alpha(q-1)) \dot{\beta} \dot{\alpha}(q-2))} \\
& +\left\{-2 d_{1}^{(q)} b_{2}^{(q)}\right\} \frac{1}{q !(q-1) !} \overline{\mathrm{D}}^{2} \mathrm{D}_{\left(\alpha_{q}\right.} \overline{\mathrm{D}}_{\left(\dot{\alpha}_{q-1}\right.} \chi_{\alpha(q-1)) \dot{\alpha}(q-2))} \\
& +\left\{-2 d_{2}^{(q)} b_{1}^{(q)}\right\} \frac{1}{q !(q-1) !} \mathrm{D}^{2} \overline{\mathrm{D}}_{\left(\dot{\alpha}_{q-1}\right.} \mathrm{D}_{\left(\alpha_{q}\right.} \chi_{\alpha(q-1)) \dot{\alpha}(q-2))} \\
& +\left\{-d_{3}^{(q)} b_{2}^{(q)}-d_{4}^{(q)}\left(b_{1}^{(q)}-\frac{q+1}{q} b_{2}^{(q)}\right)\right\} \frac{1}{q !} \mathrm{D}_{\left(\alpha_{q}\right.} \overline{\mathrm{D}}^{2} \mathrm{D}_{\alpha_{q-1}} \bar{\chi}_{\alpha(q-2)) \dot{\alpha}(q-1)} \\
& +\left\{\frac{q-1}{q} d_{4}^{(q)} b_{1}^{(q)}\right\} \frac{1}{q !(q-1) !} \mathrm{D}_{\left(\alpha_{q}\right.} \overline{\mathrm{D}}_{\left(\dot{\alpha}_{q-1}\right.} \mathrm{D}_{\alpha_{q-1}} \overline{\mathrm{D}}^{\dot{\gamma}} \bar{\chi}_{\alpha(q-2)) \dot{\gamma} \dot{\alpha}(q-1))} \\
& +\left\{\frac{q-1}{q} d_{3}^{(q)} b_{2}^{(q)}\right\} \frac{1}{q !(q-1) !} \overline{\mathrm{D}}_{\left(\dot{\alpha}_{q-1}\right.} \mathrm{D}_{\left(\alpha_{q}\right.} \overline{\mathrm{D}}^{\dot{\gamma}} \mathrm{D}_{\alpha_{q-1}} \bar{\chi}_{\alpha(q-2)) \dot{\gamma} \dot{\alpha}(q-1))} .
\end{aligned}
$$

This expression holds for all values of $q=1,2, \ldots, s-1 .{ }^{5}$ However, the validity of (3.10) can be expanded to include the $q=s$ case as well. In that case, expression (3.11) remains valid if we assign to the $d^{(s)}$ constants the corresponding values coming from the massless theory $(2.5)$

$$
d_{1}^{(s)}=0, \quad d_{2}^{(s)}=-\frac{s+1}{s}, \quad d_{3}^{(s)}=0, \quad d_{4}^{(s)}=2
$$

\footnotetext{
${ }^{5}$ Note that for $q=1$, there are no $b_{1}^{(q=1)}$ and $b_{2}^{(q=1)}$ coupling constants, as can be seen in (3.3). That is because the lowest rank $\chi$ superfield is $\chi^{(1)}$ and there is no $\chi^{(0)}$. Hence for that case the last five lines of (3.11) $\left.\right|_{q=1}$ drop out. To simplify, things we can keep using the same expression with the convention the $b_{1}^{(q=1)}=b_{2}^{(q=1)}=0$.
} 
and replace the first line with the correct $H_{\alpha(s) \dot{\alpha}(s)}$ terms

$$
\begin{aligned}
\mathcal{I}_{\alpha(s) \dot{\alpha}(s-1)}^{(s)}= & -2 m \mathrm{D}^{2} \overline{\mathrm{D}}^{\dot{\alpha}_{s}} H_{\alpha(s) \dot{\alpha}(s)} \\
& +m^{2} u_{\alpha(s) \dot{\alpha}(s-1)} \\
& +\left\{-4 d_{2}^{(s)} c_{1}^{(s)}-d_{4}^{(s)} c_{3}^{(s)}\right\} \mathrm{D}^{2} \overline{\mathrm{D}}^{2} u_{\alpha(s) \dot{\alpha}(s-1)} \\
& +\left\{-2 d_{2}^{(s)} c_{3}^{(s)}-2 d_{4}^{(s)} c_{1}^{(s)}\right\} \frac{1}{s !} \mathrm{D}^{2} \overline{\mathrm{D}}^{\dot{\alpha}_{s}} \mathrm{D}_{\left(\alpha_{s}\right.} \bar{u}_{\alpha(s-1)) \dot{\alpha}(s)} \\
& +\left\{-d_{4}^{(s)}\left(\frac{s+1}{s} c_{4}^{(s)}-c_{3}^{(s)}\right)\right\} \frac{1}{s !} \mathrm{D}_{\left(\alpha_{s}\right.} \overline{\mathrm{D}}^{2} \mathrm{D}^{\beta} u_{\beta \alpha(s-1)) \dot{\alpha}(s-1)} \\
& +\left\{-\frac{s-1}{s} d_{4}^{(s)} c_{3}^{(s)}\right\} \frac{1}{s !(s-1) !} \mathrm{D}_{\left(\alpha_{s}\right.} \overline{\mathrm{D}}_{\left(\dot{\alpha}_{s-1}\right.} \mathrm{D}^{\beta} \overline{\mathrm{D}}^{\dot{\beta}} u_{\beta \alpha(s-1)) \dot{\beta} \dot{\alpha}(s-2))} \\
& +\left\{-2 d_{2}^{(s)} b_{1}^{(s)}\right\} \frac{1}{s !(s-1) !} \mathrm{D}^{2} \overline{\mathrm{D}}_{\left(\dot{\alpha}_{s-1}\right.} \mathrm{D}_{\left(\alpha_{s}\right.} \chi_{\alpha(s-1)) \dot{\alpha}(s-2))} \\
& +\left\{-d_{4}^{(s)}\left(b_{1}^{(s)}-\frac{s+1}{s} b_{2}^{(s)}\right)\right\} \frac{1}{s !} \mathrm{D}_{\left(\alpha_{s}\right.} \overline{\mathrm{D}}^{2} \mathrm{D}_{\alpha_{s-1}} \bar{\chi}_{\alpha(s-2)) \dot{\alpha}(s-1)} \\
& +\left\{\frac{s-1}{s} d_{4}^{(s)} b_{1}^{(s)}\right\} \frac{1}{s !(s-1) !} \mathrm{D}_{\left(\alpha_{s}\right.} \overline{\mathrm{D}}_{\left(\dot{\alpha}_{s-1}\right.} \mathrm{D}_{\alpha_{s-1}} \overline{\mathrm{D}}^{\dot{\gamma}} \bar{\chi}_{\alpha(s-2)) \dot{\gamma}(s-1))}
\end{aligned}
$$

\subsection{Linearized massive supergravity and $s$-th level auxiliary superfields}

The term in the first line of (3.13) can also be generated by $\mathrm{D}^{2} \overline{\mathrm{D}}^{\dot{\alpha}_{s}} \mathcal{E}_{\alpha(s) \dot{\alpha}(s)}^{(H)}$ using the mass term of $H_{\alpha(s) \dot{\alpha}(s)}$

$$
\begin{aligned}
\mathrm{D}^{2} \overline{\mathrm{D}}^{\dot{\alpha}_{s}} \mathcal{E}_{\alpha(s) \dot{\alpha}(s)}^{(H)}= & -2 \mathrm{D}^{2} \overline{\mathrm{D}}^{2} \mathrm{D}^{2} \overline{\mathrm{D}}^{\dot{\alpha}_{s}} H_{\alpha(s) \dot{\alpha}(s)}+2 m^{2} \mathrm{D}^{2} \overline{\mathrm{D}}^{\dot{\alpha}_{s}} H_{\alpha(s) \dot{\alpha}(s)} \\
& -2 \frac{s+1}{s} \mathrm{D}^{2} \overline{\mathrm{D}}^{2} \mathrm{D}^{2} \chi_{\alpha(s) \dot{\alpha}(s-1)}+\frac{2}{s !} \mathrm{D}^{2} \overline{\mathrm{D}}^{2} \mathrm{D}_{\left(\alpha_{s}\right.} \overline{\mathrm{D}}^{\dot{\alpha}_{s}} \bar{\chi}_{\alpha(s-1)) \dot{\alpha}(s)}
\end{aligned}
$$

Unfortunately, we also generate additional, unwanted $H$ and $\chi^{(s)}$ terms. However, notice that all these additional terms can be canceled by $\mathrm{D}^{2} \overline{\mathrm{D}}^{2} \mathcal{E}_{\alpha(s) \dot{\alpha}(s-1)}^{(\chi, s)}$. If we consider the combination

$$
\begin{aligned}
\mathcal{K}_{\alpha(s) \dot{\alpha}(s-1)}^{(s)}= & \mathrm{D}^{2} \overline{\mathrm{D}}^{\dot{\alpha}(s)} \mathcal{E}_{\alpha(s) \dot{\alpha}(s)}^{(H)}+f_{1}^{(s)} \mathrm{D}^{2} \overline{\mathrm{D}}^{2} \mathcal{E}_{\alpha(s) \dot{\alpha}(s-1)}^{(\chi, s)} \\
= & \left\{-2-2 f_{1}^{(s)}\right\} \mathrm{D}^{2} \overline{\mathrm{D}}^{2} \mathrm{D}^{2} \overline{\mathrm{D}}^{\dot{\alpha}_{s}} H_{\alpha(s) \dot{\alpha}(s)} \\
& +\left\{-2 \frac{s+1}{s}-2 \frac{s+1}{s} f_{1}^{(s)}\right\} \mathrm{D}^{2} \overline{\mathrm{D}}^{2} \mathrm{D}^{2} \chi_{\alpha(s) \dot{\alpha}(s-1)} \\
& +\left\{2+2 f_{1}^{(s)}\right\} \frac{1}{s !} \mathrm{D}^{2} \overline{\mathrm{D}}^{2} \mathrm{D}_{\left(\alpha_{s}\right.} \overline{\mathrm{D}}^{\dot{\alpha}_{s}} \bar{\chi}_{\alpha(s-1)) \dot{\alpha}(s)} \\
& +2 m^{2} \mathrm{D}^{2} \overline{\mathrm{D}}^{\dot{\alpha}_{s}} H_{\alpha(s) \dot{\alpha}(s)}+f_{1}^{(s)} m \mathrm{D}^{2} \overline{\mathrm{D}}^{2} u_{\alpha(s) \dot{\alpha}(s-1)}
\end{aligned}
$$

then obviously the choice $f_{1}^{(s)}=-1$ is the appropriate one because it cancels all the unwanted terms and we are left with terms that are proportional to $m$ and $m^{2}$. These cancellations are not an accident but a consequence of the gauge invariance of the massless 
action (2.5). Because of (2.4) and (2.6) the massless theory equations of motion must satisfy the following Jacobi identity

$$
\overline{\mathrm{D}}^{\dot{\alpha}_{s}} \mathcal{E}_{\alpha(s) \dot{\alpha}(s)}^{(H, m=0)}=\overline{\mathrm{D}}^{2} \mathcal{E}_{\alpha(s) \dot{\alpha}(s-1)}^{(\chi, s, m=0)}
$$

hence, the specific combination $\mathcal{K}_{\alpha(s) \dot{\alpha}(s-1)}^{(s)}=\mathrm{D}^{2} \overline{\mathrm{D}}^{\dot{\alpha}(s)} \mathcal{E}_{\alpha(s) \dot{\alpha}(s)}^{(H)}-\mathrm{D}^{2} \overline{\mathrm{D}}^{2} \mathcal{E}_{\alpha(s) \dot{\alpha}(s-1)}^{(\chi, s)}$ must include only terms proportional to $m$ and $m^{2}$ which identically vanish in the massless limit

$$
\mathcal{K}_{\alpha(s) \dot{\alpha}(s-1)}^{(s)}=2 m^{2} \mathrm{D}^{2} \overline{\mathrm{D}}^{\dot{\alpha}_{s}} H_{\alpha(s) \dot{\alpha}(s)}-m \mathrm{D}^{2} \overline{\mathrm{D}}^{2} u_{\alpha(s) \dot{\alpha}(s-1)} .
$$

Now we can combining (3.13) with (3.17) in order to cancel the common $H$-terms

$$
\begin{aligned}
\mathcal{J}_{\alpha(s) \dot{\alpha}(s-1)}^{(s)}= & \frac{1}{m} \mathcal{K}_{\alpha(s) \dot{\alpha}(s-1)}^{(s)}+\mathcal{I}_{\alpha(s) \dot{\alpha}(s-1)}^{(s)} \\
= & m^{2} u_{\alpha(s) \dot{\alpha}(s-1)} \\
& +\left\{-4 d_{2}^{(s)} c_{1}^{(s)}-d_{4}^{(s)} c_{3}^{(s)}-1\right\} \mathrm{D}^{2} \overline{\mathrm{D}}^{2} u_{\alpha(s) \dot{\alpha}(s-1)} \\
& +\left\{-2 d_{2}^{(s)} c_{3}^{(s)}-2 d_{4}^{(s)} c_{1}^{(s)}\right\} \frac{1}{s !} \mathrm{D}^{2} \overline{\mathrm{D}}^{\dot{\alpha}_{s}} \mathrm{D}_{\left(\alpha_{s}\right.} \bar{u}_{\alpha(s-1)) \dot{\alpha}(s)} \\
& +\left\{-d_{4}^{(s)}\left(\frac{s+1}{s} c_{4}^{(s)}-c_{3}^{(s)}\right)\right\} \frac{1}{s !} \mathrm{D}_{\left(\alpha_{s}\right.} \overline{\mathrm{D}}^{2} \mathrm{D}^{\beta} u_{\beta \alpha(s-1)) \dot{\alpha}(s-1)} \\
& +\left\{-\frac{s-1}{s} d_{4}^{(s)} c_{3}^{(s)}\right\} \frac{1}{s !(s-1) !} \mathrm{D}_{\left(\alpha_{s}\right.} \overline{\mathrm{D}}_{\left(\dot{\alpha}_{s-1}\right.} \mathrm{D}^{\beta} \overline{\mathrm{D}}^{\dot{\beta}} u_{\beta \alpha(s-1)) \dot{\beta} \dot{\alpha}(s-2))} \\
& +\left\{-2 d_{2}^{(s)} b_{1}^{(s)}\right\} \frac{1}{s !(s-1) !} \mathrm{D}^{2} \overline{\mathrm{D}}_{\left(\dot{\alpha}_{s-1}\right.} \mathrm{D}_{\left(\alpha_{s}\right.} \chi_{\alpha(s-1)) \dot{\alpha}(s-2))} \\
& +\left\{-d_{4}^{(s)}\left(b_{1}^{(s)}-\frac{s+1}{s} b_{2}^{(s)}\right)\right\} \frac{1}{s !} \mathrm{D}_{\left(\alpha_{s}\right.} \overline{\mathrm{D}}^{2} \mathrm{D}_{\alpha_{s-1}} \bar{\chi}_{\alpha(s-2)) \dot{\alpha}(s-1)} \\
& +\left\{\frac{s-1}{s} d_{4}^{(s)} b_{1}^{(s)}\right\} \frac{1}{s !(s-1) !} \mathrm{D}_{\left(\alpha_{s}\right.} \overline{\mathrm{D}}_{\left(\dot{\alpha}_{s-1}\right.} \mathrm{D}_{\alpha_{s-1}} \overline{\mathrm{D}}^{\dot{\gamma}} \bar{\chi}_{\alpha(s-2)) \dot{\gamma} \dot{\alpha}(s-1))} .
\end{aligned}
$$

This gives an equation that depends only on $u^{(s)}$ and $\chi^{(s-1)}$ and includes an algebraic term for $u^{(s)}$. On-shell the left hand side of the equation vanishes, because $\mathcal{J}^{(s)}$ is constructed by equations of motions. Therefore by tuning the various coefficients we can use the algebraic term in order to make $u^{(s)}$ vanish.

For the special case of $s=1$ (linearized supergravity), the last three lines drop out and the fourth one identically vanish

$$
\begin{aligned}
\mathcal{J}_{\alpha}^{(1)}=m^{2} u_{\alpha} & +\left\{-4 d_{2}^{(1)} c_{1}^{(1)}-d_{4}^{(1)} c_{3}^{(1)}-1\right\} \mathrm{D}^{2} \overline{\mathrm{D}}^{2} u_{\alpha} \\
& +\left\{-2 d_{2}^{(1)} c_{3}^{(1)}-2 d_{4}^{(1)} c_{1}^{(1)}\right\} \mathrm{D}^{2} \overline{\mathrm{D}}^{\dot{\alpha}} \mathrm{D}_{\alpha} \bar{u}_{\dot{\alpha}} \\
& +\left\{-d_{4}^{(1)}\left(2 c_{4}^{(1)}-c_{3}^{(1)}\right)\right\} \mathrm{D}_{\alpha} \overline{\mathrm{D}}^{2} \mathrm{D}^{\beta} u_{\beta} .
\end{aligned}
$$

Therefore, in order to have $u_{\alpha}$ vanishing on-shell we must select the coefficients to satisfy

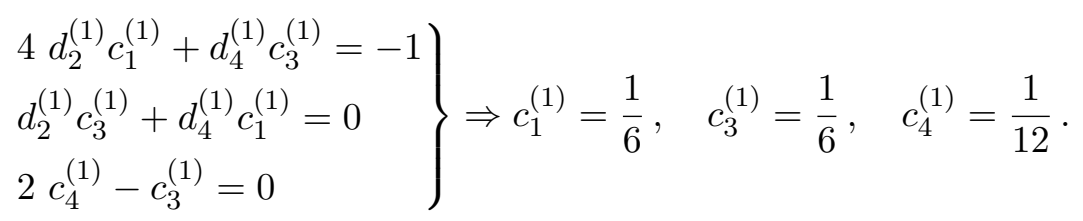


These are precisely the results found in [1].

For the general case (arbitrary $s$ ), we assume that all auxiliary superfields of lower levels vanish on-shell $u^{(r)}=\chi^{(r)}=0$ for $r=1,2, \ldots, s-1$. Then because of $\mathcal{E}_{\alpha(s-1) \dot{\alpha}(s-2)}^{(\chi, s)}=0$, superfield $u_{\alpha(s) \dot{\alpha}(s-1)}$ will satisfy the following constraint

$$
b_{1}^{(s)} \mathrm{D}^{\alpha_{s}} \overline{\mathrm{D}}^{\dot{\alpha}_{s-1}} u_{\alpha(s) \dot{\alpha}(s-1)}+b_{2}^{(s)} \overline{\mathrm{D}}^{\dot{\alpha}_{s-1}} \mathrm{D}^{\alpha_{s}} u_{\alpha(s) \dot{\alpha}(s-1)}=0
$$

which leads to the identification of various terms in (3.18)

$$
\begin{aligned}
\mathcal{J}_{\alpha(s) \dot{\alpha}(s-1)}^{(s)}= & m^{2} u_{\alpha(s) \dot{\alpha}(s-1)}+\left\{-4 d_{2}^{(s)} c_{1}^{(s)}-d_{4}^{(s)} c_{3}^{(s)}-1\right\} \mathrm{D}^{2} \overline{\mathrm{D}}^{2} u_{\alpha(s) \dot{\alpha}(s-1)} \\
& +\left\{-2 d_{2}^{(s)} c_{3}^{(s)}-2 d_{4}^{(s)} c_{1}^{(s)}\right\} \frac{1}{s !} \mathrm{D}^{2} \overline{\mathrm{D}}^{\dot{\alpha}_{s}} \mathrm{D}_{\left(\alpha_{s}\right.} \bar{u}_{\alpha(s-1)) \dot{\alpha}(s)} \\
& +\left\{-d_{4}^{(s)}\left(\frac{s+1}{s} c_{4}^{(s)}-c_{3}^{(s)}\right)-\frac{s-1}{s} d_{4}^{(s)} c_{3}^{(s)} \frac{b_{2}^{(s)}}{b_{1}^{(s)}}\right\} \frac{1}{s !} \mathrm{D}_{\left(\alpha_{s}\right.} \overline{\mathrm{D}}^{2} \mathrm{D}^{\beta} u_{\beta \alpha(s-1)) \dot{\alpha}(s-1)}
\end{aligned}
$$

Therefore, in order to be able to make $u^{(s)}$ vanish on-shell we must choose the coefficients such that

$$
\left.\begin{array}{l}
4 d_{2}^{(s)} c_{1}^{(s)}+d_{4}^{(s)} c_{3}^{(s)}=f_{1}^{(s)}=-1 \\
d_{2}^{(s)} c_{3}^{(s)}+d_{4}^{(s)} c_{1}^{(s)}=0 \\
d_{4}^{(s)}\left(\frac{s+1}{s} c_{4}^{(s)}-c_{3}^{(s)}\right)+\frac{s-1}{s} d_{4}^{(s)} c_{3}^{(s)} \frac{b_{2}^{(s)}}{b_{1}^{(s)}}=0
\end{array}\right\} \Rightarrow\left\{\begin{array}{l}
c_{1}^{(s)}=\frac{1}{4} \frac{s(s+1)}{2 s+1} \\
c_{3}^{(s)}=\frac{1}{2} \frac{s^{2}}{2 s+1} \\
c_{4}^{(s)}=\frac{1}{2} \frac{s^{3}}{(s+1)(2 s+1)}\left(1-\frac{s-1}{s} \frac{b_{2}^{(s)}}{b_{1}^{(s)}}\right)
\end{array}\right.
$$

Notice that as an input in the above system of equations are the $d^{(s)}$ and $f_{1}^{(s)}$ coefficients fixed by the massless action and Jacobi identity. This will be a repeating pattern that will become explicit in section 3.3. Moreover, $c_{2}^{(s)}$ remains arbitrary and irrelevant to $u^{(s)}=0$ which is consistent with the findings of [1], thus we can set it to zero. Also notice that the coefficients $b_{1}^{(s)}, b_{2}^{(s)}$ have not being fixed yet, however they participate in the combination $b_{2}^{(s)} / b_{1}^{(s)}$ which demands $b_{1}^{(s)}$ to be non-zero. Hence, using the freedom to normalize $\chi^{(s-1)}$ accordingly we can set it to one, $b_{1}^{(s)}=1$. Finally, the vanishing of $\chi^{(s)}$ on-shell follows automatically from equation $\mathcal{E}^{(u, s)}=0$.

\section{$3.2 Y=5 / 2$ supermultiplet and $(s-1)$-th level auxiliary superfields}

Now we repeat the process for $(s-1)$-th level auxiliary superfields. The main goal is to make $u^{(s-1)}$ vanish on-shell. If it does then, $\chi^{(s-1)}$ will automatically follow as demonstrated above. We start with (3.18) and use it to calculate $\mathrm{D}^{\alpha_{s}} \overline{\mathrm{D}}^{\dot{\alpha}_{s-1}} \mathcal{J}_{\alpha(s) \dot{\alpha}(s-1)}^{(s)}$ and $\overline{\mathrm{D}}^{\dot{\alpha}_{s-1}} \mathrm{D}^{\alpha_{s}} \mathcal{J}_{\alpha(s) \dot{\alpha}(s-1)}^{(s)}$ in order to generate the $\mathrm{D}^{\alpha_{s}} \overline{\mathrm{D}}^{\dot{\alpha}_{s-1}} u_{\alpha(s) \dot{\alpha}(s-1)}, \overline{\mathrm{D}}^{\dot{\alpha}_{s-1}} \mathrm{D}^{\alpha_{s}} u_{\alpha(s) \dot{\alpha}(s-1)}$ 
terms that appear in $I_{\alpha(s-1) \dot{\alpha}(s-2)}^{(s-1)}{ }^{6}$ After some algebra one can find that

$$
\begin{aligned}
& \mathrm{D}^{\alpha_{s}} \overline{\mathrm{D}}^{\dot{\alpha}_{s-1}} \mathcal{J}_{\alpha(s) \dot{\alpha}(s-1)}^{(s)}+b_{2}^{(s)} \overline{\mathrm{D}}^{\dot{\alpha}_{s-1}} \mathrm{D}^{\alpha_{s}} \mathcal{J}_{\alpha(s) \dot{\alpha}(s-1)}^{(s)}= \\
& =m^{2} \mathrm{D}^{\alpha_{s}} \overline{\mathrm{D}}^{\dot{\alpha}_{s-1}} u_{\alpha(s) \dot{\alpha}(s-1)}+m^{2} b_{2}^{(s)} \overline{\mathrm{D}}^{\dot{\alpha}_{s-1}} \mathrm{D}^{\alpha_{s}} u_{\alpha(s) \dot{\alpha}(s-1)} \\
& +\left\{\frac{2 s-1}{s^{2}} d_{4}^{(s)} c_{3}^{(s)}\right\} \mathrm{D}^{2} \overline{\mathrm{D}}^{2} \mathrm{D}^{\beta} \overline{\mathrm{D}}^{\dot{\beta}} u_{\beta \alpha(s-1) \dot{\beta} \dot{\alpha}(s-2)} \\
& +\left(1-\frac{s+1}{s} b_{2}^{(s)}\right)\left\{-\frac{s-1}{s} d_{4}^{(s)} c_{3}^{(s)}\right\} \mathrm{D}^{\beta} \overline{\mathrm{D}}^{2} \mathrm{D}^{2} \overline{\mathrm{D}}^{\dot{\beta}} u_{\beta \alpha(s-1) \dot{\beta} \dot{\alpha}(s-1)} \\
& +\left(1-\frac{s+1}{s} b_{2}^{(s)}\right)\left\{-\frac{s-1}{s} d_{4}^{(s)} c_{3}^{(s)} b_{2}^{(s)}\right\} \overline{\mathrm{D}}^{\dot{\beta}} \mathrm{D}^{2} \overline{\mathrm{D}}^{2} \mathrm{D}^{\beta} u_{\beta \alpha(s-1) \dot{\beta} \dot{\alpha}(s-1)} \\
& +\left\{\frac{(s-1)^{2}}{s^{2}} d_{4}^{(s)} c_{3}^{(s)} b_{2}^{(s)}\right\} \frac{1}{(s-1) !} \mathrm{D}_{\left(\alpha_{s-1}\right.} \overline{\mathrm{D}}^{2} \mathrm{D}^{\beta} \overline{\mathrm{D}}^{\dot{\gamma}} \mathrm{D}^{\gamma} u_{\beta \gamma \alpha(s-2)) \dot{\gamma} \dot{\alpha}(s-2)} \\
& +\left(1-\frac{s+1}{s} b_{2}^{(s)}\right)\left\{\frac{s-2}{s} d_{4}^{(s)} c_{3}^{(s)}\right\} \frac{1}{(s-2) !} \overline{\mathrm{D}}_{\left(\dot{\alpha}_{s-2}\right.} \mathrm{D}^{2} \overline{\mathrm{D}}^{\dot{\beta}} \mathrm{D}^{\gamma} \overline{\mathrm{D}}^{\dot{\gamma}} u_{\gamma \alpha(s-1) \dot{\beta} \dot{\gamma} \dot{\alpha}(s-3))} \\
& +\left\{-\frac{(s-2)(s-1)}{s^{2}} d_{4}^{(s)} c_{3}^{(s)}\right\} \frac{1}{(s-1) !(s-2) !} \mathrm{D}_{\left(\alpha_{s-1}\right.} \overline{\mathrm{D}}_{\left(\dot{\alpha}_{s-2}\right.} \mathrm{D}^{\beta} \overline{\mathrm{D}}^{\dot{\beta}} \mathrm{D}^{\gamma} \overline{\mathrm{D}}^{\dot{\gamma}} u_{\beta \gamma \alpha(s-2)) \dot{\beta} \dot{\gamma} \dot{\alpha}(s-3))} \\
& +\left\{2 \frac{2 s-1}{s(s-1)} d_{2}^{(s)}\right\} \mathrm{D}^{2} \overline{\mathrm{D}}^{2} \mathrm{D}^{2} \chi_{\alpha(s-1) \dot{\alpha}(s-2)} \\
& +\left\{-\frac{2 s-1}{s^{2}} d_{4}^{(s)}\right\} \frac{1}{(s-1) !} \mathrm{D}^{2} \overline{\mathrm{D}}^{2} \mathrm{D}_{\left(\alpha_{s-1}\right.} \overline{\mathrm{D}}^{\dot{\beta}} \bar{\chi}_{\alpha(s-2)) \dot{\beta} \dot{\alpha}(s-2)} \\
& +\left(1-\frac{s+1}{s} b_{2}^{(s)}\right)^{2} d_{4}^{(s)} \frac{1}{(s-1) !} \overline{\mathrm{D}}^{\dot{\beta}} \mathrm{D}^{2} \overline{\mathrm{D}}^{2} \mathrm{D}_{\left(\alpha_{s-1}\right.} \bar{\chi}_{\alpha(s-2)) \dot{\beta} \dot{\alpha}(s-2)} \\
& +\left(2 \frac{(s-1)^{2}}{s^{2}} d_{4}^{(s)}\right)\left\{1-\frac{s+1}{s-1} b_{2}^{(s)}\right\} \frac{1}{(s-1) !} \mathrm{D}_{\left(\alpha_{s-1}\right.} \overline{\mathrm{D}}^{2} \mathrm{D}^{2} \overline{\mathrm{D}}^{\dot{\beta}} \bar{\chi}_{\alpha(s-2)) \dot{\beta} \dot{\alpha}(s-2)} \\
& +\left(1-\frac{s+1}{s} b_{2}^{(s)}\right)\left\{-\frac{s-2}{s} d_{4}^{(s)}\right\} \frac{1}{(s-1) !(s-2) !} \overline{\mathrm{D}}_{\left(\dot{\alpha}_{s-2}\right.} \mathrm{D}^{2} \overline{\mathrm{D}}^{\dot{\beta}} \mathrm{D}_{\left(\alpha_{s-1}\right.} \overline{\mathrm{D}}^{\dot{\gamma}} \bar{\chi}_{\alpha(s-2)) \dot{\beta} \dot{\gamma} \dot{\alpha}(s-3))} \\
& +\left(1-\frac{s+1}{s} b_{2}^{(s)}\right)\left\{-\frac{s-2}{s} d_{4}^{(s)}\right\} \frac{1}{(s-1) !} \mathrm{D}_{\left(\alpha_{s-1}\right.} \overline{\mathrm{D}}^{2} \mathrm{D}_{\alpha_{s-2}} \overline{\mathrm{D}}^{\dot{\beta}} \mathrm{D}^{\beta} \bar{\chi}_{\beta \alpha(s-3))) \dot{\beta} \dot{\alpha}(s-2)} \\
& +\left\{-2 \frac{s-2}{s} d_{2}^{(s)}\right\} \frac{1}{(s-1) !(s-2) !} \mathrm{D}_{\left(\alpha_{s-1}\right.} \overline{\mathrm{D}}_{\left(\dot{\alpha}_{s-2}\right.} \mathrm{D}^{\beta} \overline{\mathrm{D}}^{\dot{\beta}} \mathrm{D}^{2} \chi_{\beta \alpha(s-2)) \dot{\beta} \dot{\alpha}(s-3))} \\
& +\left\{\frac{(s-2)^{2}}{s^{2}} d_{4}^{(s)}\right\} \frac{1}{(s-1) !(s-2) !} \mathrm{D}_{\left(\alpha_{s-1}\right.} \overline{\mathrm{D}}_{\left(\dot{\alpha}_{s-2}\right.} \mathrm{D}_{\alpha_{s-2}} \overline{\mathrm{D}}^{\dot{\beta}} \mathrm{D}^{\gamma} \overline{\mathrm{D}}^{\dot{\gamma}} \bar{\chi}_{\gamma \alpha(s-3)) \dot{\beta} \dot{\gamma} \dot{\alpha}(s-3))} .
\end{aligned}
$$

Once again, besides the desired terms of the first line, we generated many more $u^{(s)}$ and $\chi^{(s-1)}$ terms. However, similarly to (3.14) and (3.15), all these terms can be absorbed by appropriate use of $\mathcal{E}^{(\chi, s-1)}$ and leave only terms that depend on the mass parameter.

\footnotetext{
${ }^{6}$ That is equation (3.11) for $q=s-1$.
} 
A careful examination ofthe terms in (3.24) suggests that we have to consider the following

$$
\begin{aligned}
\mathcal{K}_{\alpha(s-1) \dot{\alpha}(s-2)}^{(s-1)}= & \mathrm{D}^{\alpha_{s}} \overline{\mathrm{D}}^{\dot{\alpha}_{s-1}} \mathcal{J}_{\alpha(s) \dot{\alpha}(s-1)}^{(s)}+b_{2}^{(s)} \overline{\mathrm{D}}^{\dot{\alpha}_{s-1}} \mathrm{D}^{\alpha_{s}} \mathcal{J}_{\alpha(s) \dot{\alpha}(s-1)}^{(s)}+f_{1}^{(s-1)} \mathrm{D}^{2} \overline{\mathrm{D}}^{2} \mathcal{E}_{\alpha(s-1) \dot{\alpha}(s-2)}^{(\chi, s-1)} \\
& +f_{2}^{(s-1)} \mathrm{D}^{\gamma} \overline{\mathrm{D}}^{2} \mathrm{D}_{\gamma} \mathcal{E}_{\alpha(s-1) \dot{\alpha}(s-2)}^{(\chi, s-1)}+\frac{f_{3}^{(s-1)}}{(s-2) !} \overline{\mathrm{D}}_{\left(\dot{\alpha}_{s-2} \mathrm{D}^{2} \overline{\mathrm{D}}^{\dot{\beta}} \mathcal{E}_{\alpha(s-1) \dot{\beta} \dot{\alpha}(s-3))}^{(\chi, s-1)}\right.} \\
& +\frac{f_{4}^{(s-1)}}{(s-1) !} \mathrm{D}_{\left(\alpha_{s-1}\right.} \overline{\mathrm{D}}^{2} \mathrm{D}^{\beta} \mathcal{E}_{\beta \alpha(s-2)) \dot{\alpha}(s-2)}^{(\chi, s-1)} \\
& +\frac{f_{5}^{(s-1)}}{(s-1) !(s-2) !} \mathrm{D}_{\left(\alpha_{s-1}\right.} \overline{\mathrm{D}}_{\left(\dot{\alpha}_{s-2}\right.} \mathrm{D}^{\beta} \overline{\mathrm{D}}^{\beta} \mathcal{E}_{\beta \alpha(s-2)) \dot{\beta} \dot{\alpha}(s-3))}^{(\chi, s-1)}
\end{aligned}
$$

for appropriate values of $f_{1}^{(s-1)}, f_{2}^{(s-1)}, f_{3}^{(s-1)}, f_{4}^{(s-1)}, f_{5}^{(s-1)}$. Notice, that many terms in (3.24) cancel if we tune $b_{2}^{(s)}$ such that

$$
1-\frac{s+1}{s} b_{2}^{(s)}=0 \Rightarrow b_{2}^{(s)}=\frac{s}{s+1} .
$$

With this choice, the contributions proportional to $f_{2}^{(s-1)}, f_{3}^{(s-1)}$ are not relevant anymore and will be ignored $\left(f_{2}^{(s-1)}=f_{3}^{(s-1)}=0\right)$

$$
\begin{aligned}
& \mathcal{K}_{\alpha(s-1) \dot{\alpha}(s-2)}^{(s-1)}= \\
& =m^{2} \mathrm{D}^{\alpha_{s}} \overline{\mathrm{D}}^{\dot{\alpha}_{s-1}} u_{\alpha(s) \dot{\alpha}(s-1)}+m^{2} b_{2}^{(s)} \overline{\mathrm{D}}^{\dot{\alpha}_{s-1}} \mathrm{D}^{\alpha_{s}} u_{\alpha(s) \dot{\alpha}(s-1)} \\
& +m f_{1}^{(s-1)} \mathrm{D}^{2} \overline{\mathrm{D}}^{2} u_{\alpha(s-1) \dot{\alpha}(s-2)}+m \frac{f_{4}^{(s-1)}}{(s-1) !} \mathrm{D}_{\left(\alpha_{s-1}\right.} \overline{\mathrm{D}}^{2} \mathrm{D}^{\beta} u_{\beta \alpha(s-2)) \dot{\alpha}(s-2)} \\
& +m \frac{f_{5}^{(s-1)}}{(s-1) !(s-2) !} \mathrm{D}_{\left(\alpha_{s-1}\right.} \overline{\mathrm{D}}_{\left(\dot{\alpha}_{s-2}\right.} \mathrm{D}^{\beta} \overline{\mathrm{D}}^{\dot{\beta}} u_{\beta \alpha(s-2)) \dot{\beta} \dot{\alpha}(s-3))} \\
& +\left\{\frac{2 s-1}{s^{2}} d_{4}^{(s)} c_{3}^{(s)}-f_{1}^{(s-1)}\right\} \mathrm{D}^{2} \overline{\mathrm{D}}^{2} \mathrm{D}^{\beta} \overline{\mathrm{D}}^{\dot{\beta}} u_{\beta \alpha(s-1) \dot{\beta} \dot{\alpha}(s-2)} \\
& +\left\{\frac{(s-1)^{2}}{s^{2}} d_{4}^{(s)} c_{3}^{(s)} b_{2}^{(s)}-f_{4}^{(s-1)} b_{2}^{(s)}\right\} \frac{1}{(s-1) !} \mathrm{D}_{\left(\alpha_{s-1}\right.} \overline{\mathrm{D}}^{2} \mathrm{D}^{\beta} \overline{\mathrm{D}}^{\dot{\gamma}} \mathrm{D}^{\gamma} u_{\beta \gamma \alpha(s-2)) \dot{\gamma} \dot{\alpha}(s-2)} \\
& +\left\{-\frac{(s-2)(s-1)}{s^{2}} d_{4}^{(s)} c_{3}^{(s)}-f_{5}^{(s-1)}\right\} \frac{1}{(s-1) !(s-2) !} \mathrm{D}_{\left(\alpha_{s-1}\right.} \overline{\mathrm{D}}_{\left(\dot{\alpha}_{s-2}\right.} \mathrm{D}^{\beta} \overline{\mathrm{D}}^{\dot{\beta}} \mathrm{D}^{\gamma} \overline{\mathrm{D}}^{\dot{\gamma}} u_{\beta \gamma \alpha(s-2)) \dot{\beta} \dot{\gamma} \dot{\alpha}(s-3))} \\
& +\left\{2 \frac{2 s-1}{s(s-1)} d_{2}^{(s)}+2 f_{1}^{(s-1)} d_{2}^{(s-1)}\right\} \mathrm{D}^{2} \overline{\mathrm{D}}^{2} \mathrm{D}^{2} \chi_{\alpha(s-1) \dot{\alpha}(s-2)} \\
& +\left\{-\frac{2 s-1}{s^{2}} d_{4}^{(s)}+f_{1}^{(s-1)} d_{4}^{(s-1)}\right\} \frac{1}{(s-1) !} \mathrm{D}^{2} \overline{\mathrm{D}}^{2} \mathrm{D}_{\left(\alpha_{s-1}\right.} \overline{\mathrm{D}}^{\dot{\beta}} \bar{\chi}_{\alpha(s-2)) \dot{\beta} \dot{\alpha}(s-2)} \\
& +\left\{\frac{(s-1)(s-2)}{s^{2}} d_{4}^{(s)}-\frac{(s+1)(s-1)}{s^{2}} d_{4}^{(s)} b_{2}^{(s)}+f_{4}^{(s-1)}\left(\frac{s}{s-1} d_{4}^{(s-1)}-d_{3}^{(s-1)}\right)+f_{5}^{(s-1)} d_{4}^{(s-1)}\right\} \times \\
& \times \frac{1}{(s-1) !} \mathrm{D}_{\left(\alpha_{s-1}\right.} \overline{\mathrm{D}}^{2} \mathrm{D}^{2} \overline{\mathrm{D}}^{\dot{\beta}} \bar{\chi}_{\alpha(s-2)) \dot{\beta} \dot{\alpha}(s-2)} \\
& +\left\{\frac{s-2}{s-1} f_{4}^{(s-1)} d_{3}^{(s-1)}\right\} \frac{1}{(s-1) !} \mathrm{D}_{\left(\alpha_{s-1}\right.} \overline{\mathrm{D}}^{2} \mathrm{D}_{\alpha_{s-2}} \overline{\mathrm{D}}^{\dot{\beta}} \mathrm{D}^{\beta} \bar{\chi}_{\beta \alpha(s-3))) \dot{\beta} \dot{\alpha}(s-2)} \\
& +\left\{-2 \frac{s-2}{s} d_{2}^{(s)}+2 f_{5}^{(s-1)} d_{2}^{(s-1)}\right\} \frac{1}{(s-1) !(s-2) !} \mathrm{D}_{\left(\alpha_{s-1}\right.} \overline{\mathrm{D}}_{\left(\dot{\alpha}_{s-2}\right.} \mathrm{D}^{\beta} \overline{\mathrm{D}}^{\dot{\beta}} \mathrm{D}^{2} \chi_{\beta \alpha(s-2)) \dot{\beta} \dot{\alpha}(s-3))} \\
& +\left\{\frac{(s-2)^{2}}{s^{2}} d_{4}^{(s)}+\frac{s-2}{s-1} f_{5}^{(s-1)} d_{4}^{(s-1)}\right\} \frac{1}{(s-1) !(s-2) !} \mathrm{D}_{\left(\alpha_{s-1}\right.} \overline{\mathrm{D}}_{\left(\dot{\alpha}_{s-2}\right.} \mathrm{D}_{\alpha_{s-2}} \overline{\mathrm{D}}^{\dot{\beta}} \mathrm{D}^{\gamma} \overline{\mathrm{D}}^{\dot{\gamma}} \bar{\chi}_{\gamma \alpha(s-3)) \dot{\beta} \dot{\gamma} \dot{\alpha}(s-3))}
\end{aligned}
$$


In order to keep only the terms that depend on mass, we must select the three $f^{(s-1)}$ in the following way

$$
\begin{aligned}
f_{1}^{(s-1)} & =\frac{2 s-1}{s^{2}} d_{4}^{(s)} c_{3}^{(s)} \\
f_{4}^{(s-1)} & =\frac{(s-1)^{2}}{s^{2}} d_{4}^{(s)} c_{3}^{(s)} \\
f_{5}^{(s-1)} & =-\frac{(s-1)(s-2)}{s^{2}} d_{4}^{(s)} c_{3}^{(s)}
\end{aligned}
$$

and the $d^{(s-1)}$ parameters must satisfy

$$
\begin{aligned}
& f_{1}^{(s-1)} d_{2}^{(s-1)}=-\frac{2 s-1}{s(s-1)} d_{2}^{(s)} \\
& f_{5}^{(s-1)} d_{2}^{(s-1)}=\frac{s-2}{s} d_{2}^{(s)} \\
& f_{1}^{(s-1)} d_{4}^{(s-1)}=\frac{2 s-1}{s^{2}} d_{4}^{(s)} \\
& f_{5}^{(s-1)} d_{4}^{(s-1)}=-\frac{(s-1)(s-2)}{s^{2}} d_{4}^{(s)} \\
& \frac{(s-1)(s-2)}{s^{2}} d_{4}^{(s)}-\frac{(s+1)(s-1)}{s^{2}} d_{4}^{(s)} b_{2}^{(s)}+f_{4}^{(s-1)}\left(\frac{s}{s-1} d_{4}^{(s-1)}-d_{3}^{(s-1)}\right)+f_{5}^{(s-1)} d_{4}^{(s-1)}=0 \\
& d_{3}^{(s)}=0
\end{aligned}
$$

Equations (3.31) are all compatible with each other and have as a solution

$$
d_{2}^{(s-1)}=-\frac{s}{s-1} \frac{d_{2}^{(s)}}{d_{4}^{(s)} c_{3}^{(s)}}, \quad d_{3}^{(s-1)}=0, \quad d_{4}^{(s-1)}=\frac{1}{c_{3}^{(s)}} .
$$

The last step is to combine $\mathcal{K}_{\alpha(s-1) \dot{\alpha}(s-2)}^{(s-1)}$ with $\mathcal{I}_{\alpha(s-1) \dot{\alpha}(s-2)}^{(s-1)}$ in order to cancel the $u^{(s)}$ term and construct $\mathcal{J}_{\alpha(s-1) \dot{\alpha}(s-2)}^{(s-1)}=\frac{1}{m} \mathcal{K}_{\alpha(s-1) \dot{\alpha}(s-2)}^{(s-1)}+\mathcal{I}_{\alpha(s-1) \dot{\alpha}(s-2)}^{(s-1)}$

$$
\begin{aligned}
& \mathcal{J}_{\alpha(s-1) \dot{\alpha}(s-2)}^{(s-1)}=m^{2} u_{\alpha(s-1) \dot{\alpha}(s-2)}+\left\{-4 d_{2}^{(s-1)} c_{1}^{(s-1)}-d_{4}^{(s-1)} c_{3}^{(s-1)}+f_{1}^{(s-1)}\right\} \mathrm{D}^{2} \overline{\mathrm{D}}^{2} u_{\alpha(s-1) \dot{\alpha}(s-2)} \\
& +\left\{-4 d_{1}^{(s-1)} c_{2}^{(s-1)}\right\} \overline{\mathrm{D}}^{2} \mathrm{D}^{2} u_{\alpha(s-1) \dot{\alpha}(s-2)}+\left\{-2 d_{1}^{(s-1)} c_{4}^{(s-1)}\right\} \frac{1}{(s-1) !} \overline{\mathrm{D}}^{2} \mathrm{D}_{\left(\alpha_{s-1}\right.} \overline{\mathrm{D}}^{\dot{\beta}} \bar{u}_{\alpha(s-2)) \dot{\beta} \dot{\alpha}(s-2)} \\
& +\left\{-2 d_{2}^{(s-1)} c_{3}^{(s-1)}-2 d_{4}^{(s-1)} c_{1}^{(s-1)}\right\} \frac{1}{(s-1) !} \mathrm{D}^{2} \overline{\mathrm{D}}^{\dot{\alpha}_{s-1}} \mathrm{D}_{\left(\alpha_{s-1}\right.} \bar{u}_{\alpha(s-2)) \dot{\alpha}(s-1)} \\
& +\left\{-d_{4}^{(s-1)}\left(\frac{s}{s-1} c_{4}^{(s-1)}-c_{3}^{(s-1)}\right)+f_{4}^{(s-1)}\right\} \frac{1}{(s-1) !} \mathrm{D}_{\left(\alpha_{s-1}\right.} \overline{\mathrm{D}}^{2} \mathrm{D}^{\beta} u_{\beta \alpha(s-2)) \dot{\alpha}(s-2)} \\
& +\left\{-\frac{s-2}{s-1} d_{4}^{(s-1)} c_{3}^{(s-1)}+f_{5}^{(s-1)}\right\} \frac{1}{(s-1) !(s-2) !} \mathrm{D}_{\left(\alpha_{s-1}\right.} \overline{\mathrm{D}}_{\left(\dot{\alpha}_{s-2}\right.} \mathrm{D}^{\beta} \overline{\mathrm{D}}^{\dot{\beta}} u_{\beta \alpha(s-2)) \dot{\beta} \dot{\alpha}(s-3))} \\
& +\left\{-2 d_{2}^{(s-1)} b_{1}^{(s-1)}\right\} \frac{1}{(s-1) !(s-2) !} \mathrm{D}^{2} \overline{\mathrm{D}}_{\left(\dot{\alpha}_{s-2}\right.} \mathrm{D}_{\left(\alpha_{s-1}\right.} \chi_{\alpha(s-2)) \dot{\alpha}(s-3))} \\
& +\left\{-2 d_{1}^{(s-1)} b_{2}^{(s-1)}\right\} \frac{1}{(s-1) !(s-2) !} \overline{\mathrm{D}}^{2} \mathrm{D}_{\left(\alpha_{s-1}\right.} \overline{\mathrm{D}}_{\left(\dot{\alpha}_{s-2}\right.} \chi_{\alpha(s-2)) \dot{\alpha}(s-3))} \\
& +\left\{-d_{4}^{(s-1)}\left(b_{1}^{(s-1)}-\frac{s}{s-1} b_{2}^{(s-1)}\right)\right\} \frac{1}{(s-1) !} \mathrm{D}_{\left(\alpha_{s-1}\right.} \overline{\mathrm{D}}^{2} \mathrm{D}_{\alpha_{s-2}} \bar{\chi}_{\alpha(s-3)) \dot{\alpha}(s-2)} \\
& +\left\{\frac{s-2}{s-1} d_{4}^{(s-1)} b_{1}^{(s-1)}\right\} \frac{1}{(s-1) !(s-2) !} \mathrm{D}_{\left(\alpha_{s-1}\right.} \overline{\mathrm{D}}_{\left(\dot{\alpha}_{s-2}\right.} \mathrm{D}_{\alpha_{s-2}} \overline{\mathrm{D}}^{\dot{\gamma}} \bar{\chi}_{\alpha(s-3)) \dot{\gamma} \dot{\alpha}(s-2))}
\end{aligned}
$$


This equation will determine all the $c^{(s-1)}$ coefficients in order to have $u^{(s-1)}$ vanishing on-shell.

For $s=2, \mathrm{Y}=5 / 2$ supermultiplet, the last four lines drop out and we are left with three equations fixing the three unknowns. For the general case, we work under the assumption that we have already set to zero all lower level auxiliary superfields $\left(u^{(r)}=\chi^{(r)}=0\right.$ for $r=1,2, \ldots, s-2)$. Therefore, using $\mathcal{E}^{(\chi, s-2)}$ we find that $u^{(s-1)}$ on-shell will satisfy the constraint

$$
b_{1}^{(s-1)} \mathrm{D}^{\alpha_{s-1}} \overline{\mathrm{D}}^{\dot{\alpha}_{s-2}} u_{\alpha(s-1) \dot{\alpha}(s-2)}+b_{2}^{(s-1)} \overline{\mathrm{D}}^{\dot{\alpha}_{s-2}} \mathrm{D}^{\alpha_{s-1}} u_{\alpha(s-1) \dot{\alpha}(s-2)}=0
$$

forcing the collapse of various terms in (3.33)

$$
\begin{gathered}
\mathcal{J}_{\alpha(s-1) \dot{\alpha}(s-2)}^{(s-1)}=m^{2} u_{\alpha(s-1) \dot{\alpha}(s-2)}+\left\{-4 d_{2}^{(s-1)} c_{1}^{(s-1)}-d_{4}^{(s-1)} c_{3}^{(s-1)}+f_{1}^{(s-1)}\right\} \mathrm{D}^{2} \overline{\mathrm{D}}^{2} u_{\alpha(q) \dot{\alpha}(q-1)} \\
+\left\{-4 d_{1}^{(s-1)} c_{2}^{(s-1)}\right\} \overline{\mathrm{D}}^{2} \mathrm{D}^{2} u_{\alpha(s-1) \dot{\alpha}(s-2)}+\left\{-2 d_{1}^{(s-1)} c_{4}^{(s-1)}\right\} \frac{1}{(s-1) !} \overline{\mathrm{D}}^{2} \mathrm{D}_{\left(\alpha_{s-1}\right.} \overline{\mathrm{D}}^{\dot{\beta}} \bar{u}_{\alpha(s-2)) \dot{\beta} \dot{\alpha}(s-2)} \\
+\left\{-2 d_{2}^{(s-1)} c_{3}^{(s-1)}-2 d_{4}^{(s-1)} c_{1}^{(s-1)}\right\} \frac{1}{(s-1) !} \mathrm{D}^{2} \overline{\mathrm{D}}^{\dot{\alpha}_{s-1}} \mathrm{D}_{\left(\alpha_{s-1}\right.} \bar{u}_{\alpha(s-2)) \dot{\alpha}(s-1)} \\
+\left\{-d_{4}^{(s-1)}\left(\frac{s}{s-1} c_{4}^{(s-1)}-c_{3}^{(s-1)}\right)+f_{4}^{(s-1)}+\frac{b_{2}^{(s-1)}}{b_{1}^{(s-1)}}\left(-\frac{s-2}{s-1} d_{4}^{(s-1)} c_{3}^{(s-1)}+f_{5}^{(s-1)}\right)\right\} \times \\
\times \frac{1}{(s-1) !} \mathrm{D}_{\left(\alpha_{s-1}\right.} \overline{\mathrm{D}}^{2} \mathrm{D}^{\beta} u_{\beta \alpha(s-2)) \dot{\alpha}(s-2)} .
\end{gathered}
$$

Hence we conclude that we must select coefficients $c_{1}^{(s-1)}, c_{3}^{(s-1)}, c_{4}^{(s-1)}$ as follows

$$
\begin{aligned}
4 d_{2}^{(s-1)} c_{1}^{(s-1)}+d_{4}^{(s-1)} c_{3}^{(s-1)} & =f_{1}^{(s-1)} \\
d_{2}^{(s-1)} c_{3}^{(s-1)}+d_{4}^{(s-1)} c_{1}^{(s-1)} & =0 \\
d_{4}^{(s-1)}\left(\frac{s}{s-1} c_{4}^{(s-1)}-c_{3}^{(s-1)}\right)+\frac{b_{2}^{(s-1)}}{b_{1}^{(s-1)}} \frac{s-2}{s-1} d_{4}^{(s-1)} c_{3}^{(s-1)} & =f_{4}^{(s-1)}+\frac{b_{2}^{(s-1)}}{b_{1}^{(s-1)}} f_{5}^{(s-1)}, \\
d_{1}^{(s-1)} c_{2}^{(s-1)} & =0 \\
d_{1}^{(s-1)} c_{4}^{(s-1)} & =0 .
\end{aligned}
$$

The solution of the above is

$$
\begin{array}{lll}
c_{1}^{(s-1)}=\frac{1}{16} \frac{(s+1) s(s-1)(2 s-1)}{(2 s+1)^{2}}, & c_{3}^{(s-1)}=-\frac{1}{8} \frac{s(s-1)^{2}(2 s-1)}{(2 s+1)^{2}}, \\
c_{4}^{(s-1)}=\frac{1}{8} \frac{(s-1)^{3}}{2 s+1}\left(1-\frac{s-2}{s-1} \frac{b_{2}^{(s-1)}}{b_{1}^{(s-1)}}\right), & d_{1}^{(s-1)}=0, \quad c_{2}^{(s-1)}=\text { arbitrary } .
\end{array}
$$

Equations (3.32) and (3.37) fix all the $(s-1)$-level coefficients. Notice that $c_{2}^{(s-1)}$ remains arbitrary and not relevant to the on-shell vanishing of $u^{(s-1)}$. Similarly to 3.1 we adopt the convention of setting it to zero $\left(c_{2}^{(s-1)}=0\right)$. However, in this case equation (3.36d) offers an explanation for this freedom. Again the input for the determination of $c^{(s-1)}$ are the $d^{(s-1)}$ and $f^{(s-1)}$ parameters. The conclusion is that the kinetic energy terms of superfield $\chi^{(s-1)}$ in (3.3) have the same structure as the kinetic energy terms of $\chi^{(s)}$ which 
are dictated by the massless limit and gauge redundancy. This has the effect of matching the kinetic energy terms of $u^{(s-1)}$ with those of $u^{(s)}$. Also, once again, coefficients $b_{2}^{(s-1)}$ and $b_{1}^{(s-1)}$ appear only in the combination $b_{2}^{(s-1)} / b_{1}^{(s-1)}$, which demands $b_{1}^{(s-1)} \neq 0$. By using the normalization of $\chi^{(s-2)}$ we can set it to one, $b_{1}^{(s-1)}=1$. Finally, the vanishing of $\chi^{(s-1)}$ trivially follows from equation $\mathcal{E}^{(u, s-1)}=0$.

\subsection{Vanishing of $q$-th level auxiliary superfields}

The above procedure can be iterated and step by step fix all coefficients such that all auxiliary superfields can be set to zero on-shell. Briefly, for the $(s-2)$ level we start with (3.33) and use it to find $\mathcal{K}^{(s-2)}$ which will include only $u^{(s-1)}$ and $u^{(s-2)}$ terms proportional to $m^{2}$ and $m$ respectively. Finding it fixes coefficients $f^{(s-2)}$ and $d^{(s-2)}$. Then we use it to find $\mathcal{J}^{(s-2)}=1 / m \mathcal{K}^{(s-2)}+\mathcal{I}^{(s-2)}$, which includes only $u^{(s-2)}$ and $\chi^{(s-3)}$ terms. Going on-shell, and assuming that $\chi^{(s-3)}$ can be set to zero ${ }^{7}$ we can choose the $c^{(s-2)}$ coefficients so $u^{(s-2)}=0$. This will also give $\chi^{(s-2)}=0$.

Here we repeat it for the arbitrary $q$-th level. We will show that there is a consistent choice of parameters such that if one assumes that all auxiliary superfields up to level $(q-1)$ vanish then $u^{(q)}$ and $\chi^{(q)}$ also vanish

$$
\left\{u^{(r)}=\chi^{(r)}=0, r=1,2, \ldots, q-1\right\} \Rightarrow u^{(q)}=0 \Rightarrow \chi^{(q)}=0 .
$$

This result holds for all values of $q=1,2, \ldots, s$ and can be used recursively. For the special case of $q=1$, our assumption $\left(u^{(r)}=\chi^{(r)}=0\right)$ is trivially satisfied because there are no such superfields, ${ }^{8}$ hence the on-shell statement $u^{(1)}=\chi^{(1)}=0$ does not rely on any hypothesis and is a pure consequence of action (3.3) given the specific values of the parameters. Hence, the assumption for the vanishing of $u^{(2)}, \chi^{(2)}$ is justified and so on for all higher auxiliary superfields. As a result, (3.38) can be applied to prove that all auxiliary superfields indeed vanish on-shell. Moreover, based on the $(s)$ and $(s-1)$ level results we conjecture that

$$
d_{1}^{(q)}=0, \quad d_{3}^{(q)}=0, \quad c_{2}^{(q)}=0, \quad b_{1}^{(q)}=1, \quad b_{2}^{(q)}=\frac{q}{q+1}, \quad \forall q=1,2, \ldots, s .
$$

Our staring point is the end result of level $(q+1)$. That means that $\mathcal{J}_{\alpha(q+1) \dot{\alpha}(q)}^{(q+1)}=1 / m \mathcal{K}_{\alpha(q+1) \dot{\alpha}(q)}^{(q+1)}+\mathcal{I}_{\alpha(q+1) \dot{\alpha}(q)}^{(q+1)}$ depends only on $u^{(q+1)}$ and $\chi^{(q)}$ superfields, $\mathcal{K}_{\alpha(q+1) \dot{\alpha}(q)}^{(q+1)}$ has only terms proportional to $m$ and $m^{2}$ of the form

$$
\begin{aligned}
& r l \mathcal{K}_{\alpha(q+1) \dot{\alpha}(q)}^{(q+1)}=m^{2} \mathrm{D}^{\alpha_{q+2}} \overline{\mathrm{D}}^{\dot{\alpha}_{q+1}} u_{\alpha(q+2) \dot{\alpha}(q+1)}+m^{2} \frac{q+2}{q+3} \overline{\mathrm{D}}^{\dot{\alpha}_{q+1}} \mathrm{D}^{\alpha_{q+2}} u_{\alpha(q+2) \dot{\alpha}(q+1)} \\
& +m f_{1}^{(q+1)} \mathrm{D}^{2} \overline{\mathrm{D}}^{2} u_{\alpha(q+1) \dot{\alpha}(q)}+m \frac{f_{4}^{(q+1)}}{(q+1) !} \mathrm{D}_{\left(\alpha_{q+1}\right.} \overline{\mathrm{D}}^{2} \mathrm{D}^{\beta} u_{\beta \alpha(q)) \dot{\alpha}(q)} \\
& +m \frac{f_{5}^{(q+1)}}{(q+1) ! q !} \mathrm{D}_{\left(\alpha_{q+1}\right.} \overline{\mathrm{D}}_{\left(\dot{\alpha}_{q}\right.} \mathrm{D}^{\beta} \overline{\mathrm{D}}^{\dot{\beta}} u_{\beta \alpha(q)) \dot{\beta} \dot{\alpha}(q-1))}
\end{aligned}
$$

\footnotetext{
${ }^{7}$ For the supermultiplet corresponding to $s=3(\mathrm{Y}=7 / 2)$ no such assumption is needed.

${ }^{8}$ Recall that we encapsulated this information in our convention to assign the following values $\left(b_{1}^{(1)}=b_{2}^{(1)}=0\right)$ for an automatic drop out of these terms.
} 
and $\mathcal{I}^{(q+1)}$ is given by (3.11) for $q \rightarrow q+1$, simplified by (3.39). The coefficients $f_{1}^{(q+1)}, f_{3}^{(q+1)}, f_{4}^{(q+1)}$ and $d_{2}^{(q+1)}, d_{4}^{(q+1)}$ have been determined by eliminating additional $u^{(q+2)}$ and $\chi^{(q+1)}$ terms. Also the coefficients $c_{1}^{(q+1)}, c_{3}^{(q+1)}, c_{4}^{(q+1)}$ have been fixes such that if $\chi^{(q)}=0$ on-shell then $\mathcal{J}_{\alpha(q+1) \dot{\alpha}(q)}^{(q+1)}=0 \Rightarrow u_{\alpha(q+1) \dot{\alpha}(q)}^{(q+1)}=0 \Rightarrow \chi_{\alpha(q+1) \dot{\alpha}(q)}^{(q+1)}=0$.

Now we use $\mathcal{J}^{(q+1)}$ together with $\mathcal{E}^{(\chi, q)}$ in order to construct $\mathcal{K}^{(q)}$

$$
\begin{aligned}
& \mathcal{K}_{\alpha(q) \dot{\alpha}(q-1)}^{(q)}=\mathrm{D}^{\alpha_{q+1}} \overline{\mathrm{D}}^{\dot{\alpha}_{q}} \mathcal{J}_{\alpha(q+1) \dot{\alpha}(q)}^{(q+1)}+\frac{q+1}{q+2} \overline{\mathrm{D}}^{\dot{\alpha}_{q}} \mathrm{D}^{\alpha_{q+1}} \mathcal{J}_{\alpha(q+1) \dot{\alpha}(q)}^{(q+1)}+f_{1}^{(q)} \mathrm{D}^{2} \overline{\mathrm{D}}^{2} \mathcal{E}_{\alpha(q) \dot{\alpha}(q-1)}^{(\chi, q)} \\
& +\frac{f_{4}^{(q)}}{q !} \mathrm{D}_{\left(\alpha_{q}\right.} \overline{\mathrm{D}}^{2} \mathrm{D}^{\beta} \mathcal{E}_{\beta \alpha(q-1)) \dot{\alpha}(q-1)}^{(\chi, q)}+\frac{f_{5}^{(q)}}{q !(q-1) !} \mathrm{D}_{\left(\alpha_{q}\right.} \overline{\mathrm{D}}_{\left(\dot{\alpha}_{q-1}\right.} \mathrm{D}^{\beta} \overline{\mathrm{D}}^{\dot{\beta}} \mathcal{E}_{\beta \alpha(q-1)) \dot{\beta} \dot{\alpha}(q-2))}^{(\chi, q)} \\
& =m^{2} \mathrm{D}^{\alpha_{q+1}} \overline{\mathrm{D}}^{\dot{\alpha}_{q}} u_{\alpha(q+1) \dot{\alpha}(q)}+m^{2} \frac{q+1}{q+2} \overline{\mathrm{D}}^{\dot{\alpha}_{q}} \mathrm{D}^{\alpha_{q+1}} u_{\alpha(q+1) \dot{\alpha}(q)}+m f_{1}^{(q)} \mathrm{D}^{2} \overline{\mathrm{D}}^{2} u_{\alpha(q) \dot{\alpha}(q-1)} \\
& +m \frac{f_{4}^{(q)}}{q !} \mathrm{D}_{\left(\alpha_{q}\right.} \overline{\mathrm{D}}^{2} \mathrm{D}^{\beta} u_{\beta \alpha(q-1)) \dot{\alpha}(q-1)}+m \frac{f_{5}^{(q)}}{q !(q-1) !} \mathrm{D}_{\left(\alpha_{q}\right.} \overline{\mathrm{D}}_{\left(\dot{\alpha}_{q-1}\right.} \mathrm{D}^{\beta} \overline{\mathrm{D}}^{\dot{\beta}} u_{\beta \alpha(q-1)) \dot{\beta} \dot{\alpha}(q-2))} \\
& +\left\{\frac{2 q+1}{(q+1)^{2}} d_{4}^{(q+1)} c_{3}^{(q+1)}-\frac{2 q+1}{q(q+1)} f_{5}^{(q+1)}-f_{1}^{(q)}\right\} \mathrm{D}^{2} \overline{\mathrm{D}}^{2} \mathrm{D}^{\beta} \overline{\mathrm{D}}^{\dot{\beta}} u_{\beta \alpha(q) \dot{\beta} \dot{\alpha}(q-1)} \\
& +\left\{\frac{q^{2}}{(q+1)(q+2)} d_{4}^{(q+1)} c_{3}^{(q+1)}-\frac{q}{q+2} f_{5}^{(q+1)}-\frac{q+1}{q+2} f_{4}^{(q)}\right\} \frac{1}{q !} \mathrm{D}_{\left(\alpha_{q}\right.} \overline{\mathrm{D}}^{2} \mathrm{D}^{\beta} \overline{\mathrm{D}}^{\dot{\gamma}} \mathrm{D}^{\gamma} u_{\beta \gamma \alpha(q-1)) \dot{\gamma} \dot{\alpha}(q-1)} \\
& +\left\{-\frac{q(q-1)}{(q+1)^{2}} d_{4}^{(q+1)} c_{3}^{(q+1)}+\frac{q-1}{q+1} f_{5}^{(q+1)}-f_{5}^{(q)}\right\} \times \\
& \times \frac{1}{q !(q-1) !} \mathrm{D}_{\left(\alpha_{q}\right.} \overline{\mathrm{D}}_{\left(\dot{\alpha}_{q-1}\right.} \mathrm{D}^{\beta} \overline{\mathrm{D}}^{\dot{\beta}} \mathrm{D}^{\gamma} \overline{\mathrm{D}}^{\dot{\gamma}} u_{\beta \gamma \alpha(q-1)) \dot{\beta} \dot{\alpha} \dot{\alpha}(q-2))} \\
& +\left\{2 \frac{2 q+1}{q(q+1)} d_{2}^{(q+1)}+2 f_{1}^{(q)} d_{2}^{(q)}\right\} \mathrm{D}^{2} \overline{\mathrm{D}}^{2} \mathrm{D}^{2} \chi_{\alpha(q) \dot{\alpha}(q-1)} \\
& +\left\{-\frac{2 q+1}{(q+1)^{2}} d_{4}^{(q+1)}+f_{1}^{(q)} d_{4}^{(q)}\right\} \frac{1}{q !} \mathrm{D}^{2} \overline{\mathrm{D}}^{2} \mathrm{D}_{\left(\alpha_{q}\right.} \overline{\mathrm{D}}^{\dot{\beta}} \bar{\chi}_{\alpha(q-1)) \dot{\beta} \dot{\alpha}(q-1)} \\
& +\left\{-\frac{q}{q+1} d_{4}^{(q+1)}+\frac{q+1}{q} f_{4}^{q)} d_{4}^{(q)}\right\} \frac{1}{q !} \mathrm{D}_{\left(\alpha_{q}\right.} \overline{\mathrm{D}}^{2} \mathrm{D}^{2} \overline{\mathrm{D}}^{\dot{\beta}} \bar{\chi}_{\alpha(q-1)) \dot{\beta} \dot{\alpha}(q-1)} \\
& +\left\{-2 \frac{q-1}{q+1} d_{2}^{(q+1)}+2 f_{5}^{(q)} d_{2}^{(q)}\right\} \frac{1}{q !(q-1) !} \mathrm{D}_{\left(\alpha_{q}\right.} \overline{\mathrm{D}}_{\left(\dot{\alpha}_{q-1}\right.} \mathrm{D}^{\beta} \overline{\mathrm{D}}^{\dot{\beta}} \mathrm{D}^{2} \chi_{\beta \alpha(q-1)) \dot{\beta} \dot{\alpha}(q-2))} \\
& +\left\{\frac{q(q-1)}{(q+1)^{2}} d_{4}^{(q+1)}+f_{5}^{(q)} d_{4}^{(q)}\right\} \frac{1}{q ! q !(q-1) !} \mathrm{D}_{\left(\alpha_{q}\right.} \overline{\mathrm{D}}_{\left(\dot{\alpha}_{q-1}\right.} \mathrm{D}^{\beta} \overline{\mathrm{D}}^{\dot{\beta}} \mathrm{D}_{(\beta} \overline{\mathrm{D}}^{\dot{\gamma}} \bar{\chi}_{\alpha(q-1))) \dot{\beta} \dot{\gamma} \dot{\alpha}(q-2))} .
\end{aligned}
$$

Demanding the cancellation of all terms that do not depend on mass fixes $f^{(q)} \mathrm{s}$ as follows

$$
\begin{aligned}
f_{1}^{(q)} & =\frac{2 q+1}{(q+1)^{2}}\left(d_{4}^{(q+1)} c_{3}^{(q+1)}-\frac{q+1}{q} f_{5}^{(q+1)}\right), \\
f_{4}^{(q)} & =\frac{q^{2}}{(q+1)^{2}}\left(d_{4}^{(q+1)} c_{3}^{(q+1)}-\frac{q+1}{q} f_{5}^{(q+1)}\right), \\
f_{5}^{(q)} & =-\frac{q(q-1)}{(q+1)^{2}}\left(d_{4}^{(q+1)} c_{3}^{(q+1)}-\frac{q+1}{q} f_{5}^{(q+1)}\right)
\end{aligned}
$$

and the $d^{(q)} \mathrm{s}$

$$
d_{2}^{(q)}=-\frac{2 q+1}{q(q+1)} \frac{d_{2}^{(q+1)}}{f_{1}^{(q)}}, \quad d_{4}^{(q)}=\frac{2 q+1}{(q+1)^{2}} \frac{d_{4}^{(q+1)}}{f_{1}^{(q)}} .
$$


The last step is to construct $\mathcal{J}^{(q)}=1 / m \mathcal{K}^{(q)}+\mathcal{I}^{(q)}$. The result is

$$
\begin{aligned}
\mathcal{J}_{\alpha(q) \dot{\alpha}(q-1)}^{(q)}= & m^{2} u_{\alpha(q) \dot{\alpha}(q-1)}+\left\{-4 d_{2}^{(q)} c_{1}^{(q)}-d_{4}^{(q)} c_{3}^{(q)}+f_{1}^{(q)}\right\} \mathrm{D}^{2} \overline{\mathrm{D}}^{2} u_{\alpha(q) \dot{\alpha}(q-1)} \\
& +\left\{-2 d_{2}^{(q)} c_{3}^{(q)}-2 d_{4}^{(q)} c_{1}^{(q)}\right\} \frac{1}{q !} \mathrm{D}^{2} \overline{\mathrm{D}}^{\dot{\beta}} \mathrm{D}_{\left(\alpha_{q}\right.} \bar{u}_{\alpha(q-1)) \dot{\beta} \dot{\alpha}(q-1)} \\
& +\left\{-d_{4}^{(q)}\left(\frac{q+1}{q} c_{4}^{(q)}-c_{3}^{(q)}\right)+f_{4}^{(q)}\right\} \frac{1}{q !} \mathrm{D}_{\left(\alpha_{q}\right.} \overline{\mathrm{D}}^{2} \mathrm{D}^{\beta} u_{\beta \alpha(q-1)) \dot{\alpha}(q-1)} \\
& +\left\{-\frac{q-1}{q} d_{4}^{(q)} c_{3}^{(q)}+f_{5}^{(q)}\right\} \frac{1}{q !(q-1) !} \mathrm{D}_{\left(\alpha_{q}\right.} \overline{\mathrm{D}}_{\left(\dot{\alpha}_{q-1}\right.} \mathrm{D}^{\beta} \overline{\mathrm{D}}^{\dot{\beta}} u_{\beta \alpha(q-1)) \dot{\beta} \dot{\alpha}(q-2))} \\
& +\left\{-2 d_{2}^{(q)}\right\} \frac{1}{q !(q-1) !} \mathrm{D}^{2} \overline{\mathrm{D}}_{\left(\dot{\alpha}_{q-1}\right.} \mathrm{D}_{\left(\alpha_{q}\right.} \chi_{\alpha(q-1)) \dot{\alpha}(q-2))} \\
& +\left\{\frac{q-1}{q} d_{4}^{(q)}\right\} \frac{1}{q !(q-1) !} \mathrm{D}_{\left(\alpha_{q}\right.} \overline{\mathrm{D}}_{\left(\dot{\alpha}_{q-1}\right.} \mathrm{D}_{\alpha_{q-1}} \overline{\mathrm{D}}^{\dot{\gamma}} \bar{\chi}_{\alpha(q-2)) \dot{\gamma} \dot{\alpha}(q-2))}
\end{aligned}
$$

The above dictates that if we want to make $u^{(q)}$ vanish on-shell, assuming $u^{(r)}=\chi^{(r)}=0,{ }^{9}$ for $r=1,2, \ldots, q-1$, we must select coefficients $c^{(q)}$ s such that:

$$
\left.\begin{array}{l}
4 d_{2}^{(q)} c_{1}^{(q)}+d_{4}^{(q)} c_{3}^{(q)}=f_{1}^{(q)} \\
d_{2}^{(q)} c_{3}^{(q)}+d_{4}^{(q)} c_{1}^{(q)}=0 \\
\frac{q+1}{q} d_{4}^{(q)} c_{4}^{(q)}-\frac{2}{q+1} d_{4}^{(q)} c_{3}^{(q)}=f_{4}^{(q)}+\frac{q}{q+1} f_{5}^{(q)}
\end{array}\right\} \Rightarrow\left\{\begin{array}{l}
c_{1}^{(q)}=-f_{1}^{(q)} \frac{d_{2}^{(q)}}{\left[d_{4}^{(q)}\right]^{2}-4\left[d_{2}^{(q)}\right]^{2}} \\
c_{3}^{(q)}=f_{1}^{(q)} \frac{d_{4}^{(q)}}{\left[d_{4}^{(q)}\right]^{2}-4\left[d_{2}^{(q)}\right]^{2}} \\
c_{4}^{(q)}=\frac{2 q}{(q+1)^{2}} c_{3}^{(q)}+\frac{q}{q+1} \frac{f_{4}^{(q)}}{d_{4}^{(q)}}+\frac{q^{2}}{(q+1)^{2}} \frac{f_{5}^{(q)}}{d_{4}^{(q)}}
\end{array}\right.
$$

\section{Off-shell degrees of freedom}

The conclusion of section 3 is that the off-shell description of an arbitrary half-integer superspin $(\mathrm{Y}=s+1 / 2)$ supermultiplet is given by the following superspace action principle

$$
\begin{aligned}
& S_{(m, \mathrm{Y}=s+1 / 2)}=\int d^{8} z\{ H^{\alpha(s) \dot{\alpha}(s)} \mathrm{D}^{\gamma} \overline{\mathrm{D}}^{2} \mathrm{D}_{\gamma} H_{\alpha(s) \dot{\alpha}(s)}+m^{2} H^{\alpha(s) \dot{\alpha}(s)} H_{\alpha(s) \dot{\alpha}(s)} \\
&-2 H^{\alpha(s) \dot{\alpha}(s)} \overline{\mathrm{D}}_{\dot{\alpha}_{s}} \mathrm{D}^{2} \chi_{\alpha(s) \dot{\alpha}(s-1)}+c . c . \\
&+\sum_{q=1}^{s}\left[d_{2}^{(q)} \chi^{\alpha(q) \dot{\alpha}(q-1)} \mathrm{D}^{2} \chi_{\alpha(q) \dot{\alpha}(q-1)}+c_{1}^{(q)} u^{\alpha(q) \dot{\alpha}(q-1)} \overline{\mathrm{D}}^{2} u_{\alpha(q) \dot{\alpha}(q-1)}+\right.\text { c.c. } \\
& \quad+d_{4}^{(q)} \chi^{\alpha(q) \dot{\alpha}(q-1)} \mathrm{D}_{\alpha_{q}} \overline{\mathrm{D}}^{\dot{\alpha}_{q}} \bar{\chi}_{\alpha(q-1) \dot{\alpha}(q)}+c_{3}^{(q)} u^{\alpha(q) \dot{\alpha}(q-1)} \overline{\mathrm{D}}^{\dot{\alpha}_{q}} \mathrm{D}_{\alpha_{q}} \bar{u}_{\alpha(q-1) \dot{\alpha}(q)} \\
&\left.\quad+c_{4}^{(q)} u^{\alpha(q) \dot{\alpha}(q-1)} \mathrm{D}_{\alpha_{q}} \overline{\mathrm{D}}^{\dot{\alpha}_{q}} \bar{u}_{\alpha(q-1) \dot{\alpha}(q)}+m\left(\chi^{\alpha(q) \dot{\alpha}(q-1)} u_{\alpha(q) \dot{\alpha}(q-1)}+\text { c.c. }\right)\right] \\
&\left.+\sum_{q=1}^{s-1}\left[u^{\alpha(q+1) \dot{\alpha}(q)}\left(\overline{\mathrm{D}}_{\dot{\alpha}_{q}} \mathrm{D}_{\alpha_{q+1}} \chi_{\alpha(q) \dot{\alpha}(q-1)}+\frac{q+1}{q+2} \mathrm{D}_{\alpha_{q+1}} \overline{\mathrm{D}}_{\dot{\alpha}_{q}} \chi_{\alpha(q) \dot{\alpha}(q-1)}\right)+\text { c.c. }\right]\right\}
\end{aligned}
$$

\footnotetext{
${ }^{9}$ Besides dropping the last two terms of (3.44), it also imposes on $u^{(q)}$ the constraint $\mathrm{D}^{\beta} \overline{\mathrm{D}}^{\dot{\beta}} u_{\beta \alpha(q-1) \dot{\beta} \dot{\alpha}(q-2)}+\frac{q}{q+1} \overline{\mathrm{D}}^{\dot{\beta}} \mathrm{D}^{\beta} u_{\beta \alpha(q-1) \dot{\beta} \dot{\alpha}(q-2)}=0$.
} 
where the coefficients $d^{(q)}$ and $c^{(q)}$ are given by the recursive relations (3.42), (3.43) and (3.45) together with the initial conditions fixed by the massless limit of the theory

$$
d_{2}^{(s)}=-\frac{s+1}{s}, \quad d_{4}^{(s)}=2, \quad f_{1}^{(s)}=-1, \quad f_{4}^{(s)}=f_{5}^{(s)}=0 .
$$

The off-shell structure of this theory is extremely rich, since it requires the presence of two towers of auxiliary superfields with $s$ members each of increasing rank $\left\{u^{(r)}, \chi^{(r)}\right\} r=1,2, \ldots, s$. By projecting the superspace action to components, we can find the component structure of the theory. It will include the auxiliary fields required by $[23,24]$ for the off-shell description of irreducible, arbitrary higher spins plus additional auxiliary fields required by off-shell supersymmetry. Because all participating superfields are unconstrained and there is no redundancy, all the components of every superfield will participate in the off-shell component action.

It is straightforward to count the off-shell degrees of freedom of an unconstrained $(n, m)$ superfield $\Phi_{\alpha(n) \dot{\alpha}(m)}$. The answer is $16(n+1)(m+1)$ bosons ${ }^{10}$ and equal number of fermions. An exception is the case were we can impose a reality condition $(\Phi=\bar{\Phi} \Rightarrow n=m)$. In that case, the real superfield $\Phi_{\alpha(n) \dot{\alpha}(n)}$ carries $8(n+1)^{2}$ bosons and equally many fermions. Therefore the total number of off-shell degrees of freedom of this theory is

$$
8(s+1)^{2}+2 \times \sum_{n=1}^{s} 16(n+1) n=\frac{8}{3}(s+1)\left(4 s^{2}+11 s+3\right) .
$$

\section{Summary}

The supersymmetric Fierz-Pauli program of constructing superspace Lagrangians for higher spin supermultiplets has been a long standing question since the birth of supersymmetry. In this paper we answer this question for arbitrary half-integer $(\mathrm{Y}=s+1 / 2)$ supermultiplets that on-shell describe the propagation of free massive spins $j=s+1, j=s+1 / 2, j=s+1 / 2, j=s$. We find that the off-shell superspace action description of this supermultiplet requires a tower of pairs of auxiliary superfields $u_{\alpha(q) \dot{\alpha}(q-1)}, \chi_{\alpha(q) \dot{\alpha}(q-1)}$ with $q=1,2, \ldots, s$ and it has the form (4.1).

The coupling constants $d_{2}^{(q)}, d_{4}^{(q)}$ and $c_{1}^{(q)}, c_{3}^{(q)}, c_{4}^{(q)}$ of $(4.1)$ are given by the recursive relations (3.42), (3.43) and (3.45) with initial conditions (4.2). They have been determined such that on-shell $(i)$ all auxiliary superfields vanish $\left(u_{\alpha(q) \dot{\alpha}(q-1)}=\chi_{\alpha(q) \dot{\alpha}(q-1)}=0\right)(i i)$ we generate the appropriate constraints for superfield $H_{\alpha(s) \dot{\alpha}(s)}\left(\mathrm{D}^{\alpha_{s}} H_{\alpha(s) \dot{\alpha}(s)}=0, \square H_{\alpha(s) \dot{\alpha}(s)}=m^{2} H_{\alpha(s) \dot{\alpha}(s)}\right)$ which allow only the above mentioned spin degrees of freedom to propagate and ( $i i i)$ the massless limit of the action gives a smooth transition to the correct description of the arbitrary massless half-integer superspin supermultiplet by decoupling all auxiliary superfields with the exception of $\chi_{\alpha(s) \dot{\alpha}(s-1)}$ which becomes the compensator of $H_{\alpha(s) \dot{\alpha}(s)}$, as required by the gauge redundancy of the massless theory.

\footnotetext{
${ }^{10}$ For details see [42].
} 
These recursion relations can be iterated systematically to extract the numerical value of all coefficients. For example, as demonstrated by (3.23), (3.37) and (4.2), (3.32), (3.39) the first two levels of coefficients, for arbitrary values of the parameter $s$, are:

$$
\begin{array}{rlrl}
c_{1}^{(s)} & =\frac{1}{4} \frac{s(s+1)}{2 s+1}, \quad c_{3}^{(s)}=\frac{1}{2} \frac{s^{2}}{2 s+1}, \quad c_{4}^{(s)} & =\frac{s^{3}}{(s+1)^{2}(2 s+1)}, \quad d_{2}^{(s)}=-\frac{s+1}{s}, \quad d_{4}^{(s)}=2 \\
c_{1}^{(s-1)} & =\frac{1}{16} \frac{(s+1) s(s-1)(2 s-1)}{(2 s+1)^{2}}, & c_{3}^{(s-1)} & =-\frac{1}{8} \frac{s(s-1)^{2}(2 s-1)}{(2 s+1)^{2}}, \\
c_{4}^{(s-1)} & =\frac{1}{4} \frac{(s-1)^{3}}{s(2 s+1)}, & d_{2}^{(s-1)} & =\frac{(s+1)(2 s+1)}{s^{2}(2 s+1)}, \quad d_{4}^{(s-1)}=2 \frac{2 s+1}{s^{2}}
\end{array}
$$

For the special case of $s=1,(5.1)$ give the numerical value of the coefficients required for the description of massive $Y=3 / 2$ supermultiplet and they are in agreement with the findings of [1]. For $s=2$ (5.1), (5.2) give the numerical value of coefficients required for the description of massive $Y=5 / 2$ supermultiplet.

Moreover, the coefficients $d_{1}^{(q)}$ and $d_{3}^{(q)}$ vanish for all values of $q$. For $q=s$ this is a consequence of the gauge invariance of the massless action and it's effect trickles down to all other levels. As a result $c_{2}^{(q)}$ drops out of all equations (for example (3.36d)), remains undetermined and not relevant for the on-shell spectrum of the theory. For simplicity we set it to zero.

A characteristic feature of the theory is that it requires the presence of the 'bare' superfield $\chi_{\alpha(s) \dot{\alpha}(s-1)}$. This means that the constrained superfield $\left(\Gamma_{\alpha(s-1) \dot{\alpha}(s-1)} \propto \overline{\mathrm{D}}^{\dot{\alpha}_{s}} \bar{\chi}_{\alpha(s-1) \dot{\alpha}(s)}\right)$ approach of $[6,7]$ can not be used to generate this result. From the view point of the massive theory the unconstrained superfield approach of $[9,10]$ seem to be the more appropriate variables that one should consider.

Due to the plethora of the auxiliary superfields, the off-shell structure of the theory is extremely rich and the number of off-shell degrees of freedom scale as $\sim s^{3}$. Specifically, the theory carries $\frac{8}{3}(s+1)\left(4 s^{2}+11 s+3\right)$ bosons and equal number of fermions. In contrast, the on-shell degrees of freedom are just the $4(s+1)$ polarizations of bosonic spins $j=s+1, j=s$ and an equal number of states coming from the two $j=s+1 / 2$ fermionic spins.

\section{Acknowledgments}

This work is supported in part by S. James Gates Jr.'s endowment of the Ford Foundation Professorship of Physics at Brown University and the author gratefully acknowledge the support of the Brown Theoretical Physics Center. 
Open Access. This article is distributed under the terms of the Creative Commons Attribution License (CC-BY 4.0), which permits any use, distribution and reproduction in any medium, provided the original author(s) and source are credited.

\section{References}

[1] S.J. Gates Jr. and K. Koutrolikos, A dynamical theory for linearized massive superspin 3/2, JHEP 03 (2014) 030 [arXiv: 1310.7387] [inSPIRE].

[2] G.L. Kane and M. Shifman eds., The supersymmetric world: The beginning of the theory, World Scientific (2000) [INSPIRE].

[3] M.A. Shifman ed., The many faces of the superworld: Yuri Golfand memorial volume, Singapore (2000) [INSPIRE].

[4] T. Curtright, Massless Field Supermultiplets With Arbitrary Spin, Phys. Lett. B 85 (1979) 219 [INSPIRE].

[5] M.A. Vasiliev, 'Gauge' form of description of massless fields with arbitrary spin (in Russian), Yad. Fiz. 32 (1980) 855 [INSPIRE].

[6] S.M. Kuzenko, A.G. Sibiryakov and V.V. Postnikov, Massless gauge superfields of higher half integer superspins, JETP Lett. 57 (1993) 534 [INSPIRE].

[7] S.M. Kuzenko and A.G. Sibiryakov, Massless gauge superfields of higher integer superspins, JETP Lett. 57 (1993) 539 [INSPIRE].

[8] S.M. Kuzenko and A.G. Sibiryakov, Free massless higher superspin superfields on the anti-de Sitter superspace, Phys. Atom. Nucl. 57 (1994) 1257 [arXiv:1112.4612] [INSPIRE].

[9] S.J. Gates Jr. and K. Koutrolikos, On $4 D, \mathcal{N}=1$ massless gauge superfields of arbitrary superhelicity, JHEP 06 (2014) 098 [arXiv: 1310.7385] [INSPIRE].

[10] S.J. Gates Jr. and K. Koutrolikos, On 4D, $N=1$ Massless Gauge Superfields of Higher Superspin: Half-Odd-Integer Case, arXiv:1310.7386 [INSPIRE].

[11] I.L. Buchbinder, S.J. Gates and K. Koutrolikos, Hierarchy of Supersymmetric Higher Spin Connections, Phys. Rev. D 102 (2020) 125018 [arXiv: 2010.02061] [INSPIRE].

[12] I.L. Buchbinder, S.J. Gates and K. Koutrolikos, Higher Spin Superfield interactions with the Chiral Supermultiplet: Conserved Supercurrents and Cubic Vertices, Universe 4 (2018) 6 [arXiv: 1708.06262] [INSPIRE].

[13] J. Hutomo and S.M. Kuzenko, Non-conformal higher spin supercurrents, Phys. Lett. B 778 (2018) 242 [arXiv: 1710.10837] [INSPIRE].

[14] K. Koutrolikos, P. Kočí and R. von Unge, Higher Spin Superfield interactions with Complex linear Supermultiplet: Conserved Supercurrents and Cubic Vertices, JHEP 03 (2018) 119 [arXiv: 1712.05150] [INSPIRE].

[15] I.L. Buchbinder, S.J. Gates and K. Koutrolikos, Interaction of supersymmetric nonlinear sigma models with external higher spin superfields via higher spin supercurrents, JHEP 05 (2018) 204 [arXiv : 1804.08539] [InSPIRE].

[16] I.L. Buchbinder, S.J. Gates and K. Koutrolikos, Conserved higher spin supercurrents for arbitrary spin massless supermultiplets and higher spin superfield cubic interactions, JHEP 08 (2018) 055 [arXiv: 1805. 04413] [INSPIRE]. 
[17] E.I. Buchbinder, J. Hutomo and S.M. Kuzenko, Higher spin supercurrents in anti-de Sitter space, JHEP 09 (2018) 027 [arXiv: 1805.08055] [INSPIRE].

[18] I.L. Buchbinder, S.J. Gates and K. Koutrolikos, Integer superspin supercurrents of matter supermultiplets, JHEP 05 (2019) 031 [arXiv:1811.12858] [INSPIRE].

[19] S.J. Gates and K. Koutrolikos, Progress on cubic interactions of arbitrary superspin supermultiplets via gauge invariant supercurrents, Phys. Lett. B 797 (2019) 134868 [arXiv: 1904.13336] [INSPIRE].

[20] R.R. Metsaev, Cubic interaction vertices for $N=1$ arbitrary spin massless supermultiplets in flat space, JHEP 08 (2019) 130 [arXiv: 1905.11357] [INSPIRE].

[21] R.R. Metsaev, Cubic interactions for arbitrary spin $\mathcal{N}$-extended massless supermultiplets in 4d flat space, JHEP 11 (2019) 084 [arXiv: 1909.05241] [INSPIRE].

[22] M.V. Khabarov and Y.M. Zinoviev, Cubic interaction vertices for massless higher spin supermultiplets in $d=4$, JHEP 02 (2021) 167 [arXiv: 2012.00482] [INSPIRE].

[23] L.P.S. Singh and C.R. Hagen, Lagrangian formulation for arbitrary spin. 1. The boson case, Phys. Rev. D 9 (1974) 898 [inSPIRE].

[24] L.P.S. Singh and C.R. Hagen, Lagrangian formulation for arbitrary spin. 2. The fermion case, Phys. Rev. D 9 (1974) 910 [InSPIRE].

[25] P.A. Dirac, Relativistic wave equations, Proc. Roy. Soc. Lond. A 155 (1936) 447.

[26] Y.M. Zinoviev, Massive $N=1$ supermultiplets with arbitrary superspins, Nucl. Phys. B $\mathbf{7 8 5}$ (2007) 98 [arXiv:0704.1535] [INSPIRE].

[27] I.L. Buchbinder, M.V. Khabarov, T.V. Snegirev and Y.M. Zinoviev, Lagrangian formulation of the massive higher spin $N=1$ supermultiplets in $A d S_{4}$ space, Nucl. Phys. B 942 (2019) 1 [arXiv: 1901.09637] [INSPIRE].

[28] M.V. Khabarov and Y.M. Zinoviev, Massive higher spin fields in the frame-like multispinor formalism, Nucl. Phys. B 948 (2019) 114773 [arXiv:1906.03438] [InSPIRE].

[29] Y.M. Zinoviev, Massive Higher Spins in Multispinor Formalism, Phys. Part. Nucl. Lett. 17 (2020) 692 [INSPIRE].

[30] M.V. Khabarov and Y.M. Zinoviev, Massive higher spin supermultiplets unfolded, Nucl. Phys. B 953 (2020) 114959 [arXiv:2001.07903] [InSPIRE].

[31] I.L. Buchbinder, S.J. Gates Jr., W.D. Linch, III and J. Phillips, New 4-D, $N=1$ superfield theory: Model of free massive superspin 3/2 multiplet, Phys. Lett. B $\mathbf{5 3 5}$ (2002) 280 [hep-th/0201096] [INSPIRE].

[32] I.L. Buchbinder, S.J. Gates Jr., W.D. Linch, III and J. Phillips, Dynamical superfield theory of free massive superspin-1 multiplet, Phys. Lett. B 549 (2002) 229 [hep-th/0207243] [INSPIRE].

[33] T. Gregoire, M.D. Schwartz and Y. Shadmi, Massive supergravity and deconstruction, JHEP 07 (2004) 029 [hep-th/0403224] [INSPIRE].

[34] I.L. Buchbinder, S. James Gates Jr., S.M. Kuzenko and J. Phillips, Massive $4 D, N=1$ superspin 1 \& 3/2 multiplets and dualities, JHEP 02 (2005) 056 [hep-th/0501199] [INSPIRE].

[35] S.J. Gates Jr., S.M. Kuzenko and G. Tartaglino-Mazzucchelli, New massive supergravity multiplets, JHEP 02 (2007) 052 [hep-th/0610333] [INSPIRE]. 
[36] I. Bars, Supergroups and Their Representations, Lectures Appl. Math. 21 (1983) 17 [inSPIRE].

[37] S.J. Gates, M.T. Grisaru, M. Roček and W. Siegel, Superspace Or One Thousand and One Lessons in Supersymmetry, Frontiers in Physics 58 (1983) [hep-th/0108200] [INSPIRE].

[38] P. Srivastava, Supersymmetry, superfields and supergravity: an introduction, Bristol, U.K. (1986).

[39] I. Buchbinder and S. Kuzenko, Ideas and methods of supersymmetry and supergravity: A Walk through superspace, Bristol, U.K. (1995).

[40] K. Koutrolikos, On Lagrangian Formulation of Higher-Superspin Irreducible Representations of the Super-Poincaré Group, Ph.D. Thesis, Maryland University, Maryland, U.S.A. (2013).

[41] I.L. Buchbinder, S.J. Gates and K. Koutrolikos, Superfield continuous spin equations of motion, Phys. Lett. B 793 (2019) 445 [arXiv: 1903.08631] [INSPIRE].

[42] S.J. Gates and K. Koutrolikos, From Diophantus to Supergravity and massless higher spin multiplets, JHEP 11 (2017) 063 [arXiv:1707.00194] [INSPIRE]. 